\title{
Cubical Convex Ear Decompositions
}

\author{
Russ Woodroofe \\ Department of Mathematics \\ Washington University in St. Louis \\ St. Louis, MO 63130, USA \\ russw@math . wustl .edu
}

Submitted: Aug 8, 2008; Accepted: Jun 5, 2009; Published: Jun 10, 2009

Mathematics Subject Classification: 05E25

Dedicated to Anders Björner in honor of his 60th birthday.

\begin{abstract}
We consider the problem of constructing a convex ear decomposition for a poset. The usual technique, introduced by Nyman and Swartz, starts with a $C L$-labeling and uses this to shell the 'ears' of the decomposition. We axiomatize the necessary conditions for this technique as a " $C L$-ced" or " $E L$-ced". We find an $E L$-ced of the $d$-divisible partition lattice, and a closely related convex ear decomposition of the coset lattice of a relatively complemented finite group. Along the way, we construct new EL-labelings of both lattices. The convex ear decompositions so constructed are formed by face lattices of hypercubes.

We then proceed to show that if two posets $P_{1}$ and $P_{2}$ have convex ear decompositions $\left(C L\right.$-ceds), then their products $P_{1} \times P_{2}, P_{1} \check{\times} P_{2}$, and $P_{1} \hat{\times} P_{2}$ also have convex ear decompositions ( $C L$-ceds). An interesting special case is: if $P_{1}$ and $P_{2}$ have polytopal order complexes, then so do their products.
\end{abstract}

\section{Contents}

1 Introduction $\quad 2$

2 Definitions and tools $\quad 3$

2.1 Convex ear decompositions . . . . . . . . . . . . . . . . 4

2.2 Shellings . . . . . . . . . . . . . . . . . . . . . 4

2.3 Supersolvable lattices . . . . . . . . . . . . . . . . . . . . . . . . 5

2.4 Cohen-Macaulay complexes . . . . . . . . . . . . . . . . 6

$2.5 \quad E L$-ceds and $C L$-ceds . . . . . . . . . . . . . . . . . . . . . . . 7 
3 The $d$-divisible partition lattice $\quad \mathbf{9}$

$3.1 \quad$ A dual $E L$-labeling for $\Pi_{n}^{d} \ldots \ldots \ldots \ldots$

3.2 An $E L$-ced for $\Pi_{n}^{d} \ldots \ldots \ldots \ldots \ldots$

4 The coset lattice $\quad 17$

4.1 Group theory background $\ldots \ldots \ldots \ldots \ldots \ldots$

$4.2 \quad$ A dual $E L$-labeling for $\mathfrak{C}(G) \ldots \ldots \ldots \ldots$

4.3 A convex ear decomposition for $\mathfrak{C}(G) \ldots \ldots \ldots \ldots$

5 Poset products 24

5.1 Poset products and polytopes . . . . . . . . . . . . . . . 24

5.2 Convex ear decompositions of product posets . . . . . . . . . . . . . 27

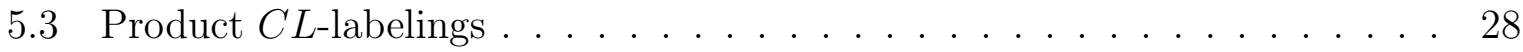

$5.4 C L$-ceds of product posets . . . . . . . . . . . . . . . . . 29

6 Further questions $\quad 30$

\section{Introduction}

Convex ear decompositions, introduced by Chari in [6], break a simplicial complex into subcomplexes of convex polytopes in a manner with nice properties for enumeration. A complex with a convex ear decomposition inherits many properties of convex polytopes. For example, such a complex has a unimodal $h$-vector [6], with an analogue of the $g$ theorem holding [31], and is doubly Cohen-Macaulay [31].

Nyman and Swartz constructed a convex ear decomposition for geometric lattices in [17]. Their proof method used the EL-labeling of such lattices to understand the decomposition's topology. Similar techniques were pushed further by Schweig [23]. In Section 2, we introduce the necessary background material, and then axiomatize the conditions necessary for these techniques. We call such a convex ear decomposition a "CL-ced", or " $E L$-ced."

We then show by example in Sections 3 and 4 how to use these techniques on some poset families: $d$-divisible partition lattices, and coset lattices of a relatively complemented group. These posets have each interval $[a, \hat{1}]$ supersolvable, where $a \neq \hat{0}$. Finding the convex ear decompositions will involve constructing a (dual) EL-labeling that respects the supersolvable structure up to sign, and showing that a set of (barycentricly subdivided) hypercubes related to the $E L$-labeling is an $E L$-ced, or at least a convex ear decomposition. We will prove specifically:

Theorem 1.1. The d-divisible partition lattice $\Pi_{n}^{d}$ has an EL-ced, hence a convex ear decomposition.

Theorem 1.2. The coset lattice $\mathfrak{C}(G)$ has a convex ear decomposition if and only if $G$ is a relatively complemented finite group. 
I believe these convex ear decompositions to be the first large class of examples where each ear is a hypercube.

Although both poset families were known to be $E L$-shellable, the $E L$-labelings that we construct in these sections also seem to be new. The ideas used to find them may be applicable in other settings, as briefly discussed in Section 6.

Lemma 1.3. $\Pi_{n}^{d}$ has a dual EL-labeling; $\mathfrak{C}(G)$ has a dual EL-labeling if $G$ is a complemented finite group.

In Section 5 we change focus slightly to discuss products of bounded posets. Our first goal is:

Theorem 1.4. If bounded posets $P_{1}$ and $P_{2}$ have convex ear decompositions, then so do $P_{1} \times P_{2}, P_{1} \check{\times} P_{2}$, and $P_{1} \hat{\times} P_{2}$.

This is the first result of which I am aware that links poset constructions and convex ear decompositions with such generality. A result of a similar flavor (but more restrictive) is proved by Schweig [23]: that rank selected subposets of some specific families of posets have convex ear decompositions.

A special case of Theorem 1.4 has a particularly pleasing form:

Lemma 1.5. If $P_{1}$ and $P_{2}$ are bounded posets such that $\left|P_{1}\right|$ and $\left|P_{2}\right|$ are isomorphic to the boundary complexes of simplicial polytopes, then so are $\left|P_{1} \times P_{2}\right|,\left|P_{1} \times P_{2}\right|$, and $\left|P_{1} \hat{\times} P_{2}\right|$.

We then recall the work of Björner and Wachs [4, Section 10] on $C L$-labelings of poset products, which we use to prove a result closely related to Theorem 1.4:

Theorem 1.6. If bounded posets $P_{1}$ and $P_{2}$ have $C L$-ceds with respect to $C L$-labelings $\lambda_{1}$ and $\lambda_{2}$, then $P_{1} \times P_{2}, P_{1} \check{\times} P_{2}$, and $P_{1} \hat{\times} P_{2}$ have $C L$-ceds with respect to the labelings $\lambda_{1} \times \lambda_{2}, \lambda_{1} \check{\times} \lambda_{2}$, and $\lambda_{1} \hat{\times} \lambda_{2}$.

We close by considering some additional questions and directions for further research in Section 6.

\section{Definitions and tools}

All simplicial complexes, posets, and groups discussed in this paper are finite.

A poset $P$ is bounded if it has a lower bound $\hat{0}$ and an upper bound $\hat{1}$, so that $\hat{0} \leq x \leq \hat{1}$ for all $x \in P$.

If $P$ is a bounded poset, then the order complex $|P|$ is the simplicial complex whose faces are the chains of $P \backslash\{\hat{0}, \hat{1}\}$. (This is slightly different from the standard definition, in that we are taking only the proper part of the poset.) Where it will cause no confusion, we talk about $P$ and $|P|$ interchangeably: for example, we say $P$ has a convex ear decomposition if $|P|$ does.

We denote by $\mathcal{M}(P)$ the set of maximal chains of $P$, which is in natural bijective correspondence with the facets of $|P|$ through adding or removing $\hat{0}$ and $\hat{1}$. 


\subsection{Convex ear decompositions}

A convex ear decomposition of a pure $(d-1)$-dimensional simplicial complex $\Delta$ is an ordered collection of subcomplexes $\Delta_{1}, \ldots, \Delta_{m} \subseteq \Delta$ with the following properties:

ced-polytope $\Delta_{s}$ is isomorphic to a subcomplex of the boundary complex of a simplicial $d$-polytope for each $s$.

ced-topology $\Delta_{1}$ is a $(d-1)$-sphere, and $\Delta_{s}$ is a $(d-1)$-ball for $s>1$.

ced-bdry $\left(\bigcup_{t=1}^{s-1} \Delta_{t}\right) \cap \Delta_{s}=\partial \Delta_{s}$ for each $s>1$.

ced-union $\bigcup_{s=1}^{m} \Delta_{s}=\Delta$.

It follows immediately from the definition that any complex with a convex ear decomposition is pure. As far as I know, no one has tried generalizing the theory of convex ear decompositions to non-pure complexes. As many interesting posets are not graded (i.e., have an order complex that is not pure), finding such a generalization could be useful.

Convex ear decompositions were first introduced by Chari [6]. He used the unimodality of the $h$-vector of a simplicial polytope to give a strong condition on the $h$-vector for a complex with a convex ear decomposition. Swartz [31] showed that a ' $g$-theorem' holds for any $(d-1)$-dimensional complex with a convex ear decomposition, as stated precisely in Theorem 2.2. We refer the reader to [28] for further background on $h$-vectors, $M$-vectors, and the (original) $g$-theorem.

Theorem 2.1. (Chari [6, Section 3]) The h-vector of a pure (d-1)-dimensional complex with a convex ear decomposition satisfies the conditions

$$
\begin{gathered}
h_{0} \leq h_{1} \leq \cdots \leq h_{\lfloor d / 2\rfloor} \\
h_{i} \leq h_{d-i}, \quad \text { for } 0 \leq i \leq\lfloor d / 2\rfloor .
\end{gathered}
$$

Theorem 2.2. (Swartz [31, Corollary 3.10]) If $\left\{h_{i}\right\}$ is the $h$-vector of a pure $(d-1)$ dimensional complex with a convex ear decomposition, then

$$
\left(h_{0}, h_{1}-h_{0}, \ldots, h_{\lfloor d / 2\rfloor}-h_{\lfloor d / 2\rfloor-1}\right)
$$

is an $M$-vector.

\subsection{Shellings}

An essential tool for us will be the theory of lexicographic shellability, developed by Björner and Wachs in $[1,2,3,4]$. We recall some of the main facts.

We say that an ordering of the facets $F_{1}, F_{2}, \ldots, F_{t}$ of a simplicial complex $\Delta$ (with $t$ facets) is a shelling if $F_{i} \cap\left(\bigcup_{j=1}^{i-1} F_{j}\right)$ is pure $\left(\operatorname{dim} F_{i}-1\right)$-dimensional for all $1<i \leq t$. An equivalent condition that is often easier to use is:

$$
\begin{aligned}
& \text { if } 1 \leq i<j \leq t \text {, then } \exists k<j \text { such that } \\
& F_{i} \cap F_{j} \subseteq F_{k} \cap F_{j}=F_{j} \backslash\{x\} \text { for some } x \in F_{j} \text {. }
\end{aligned}
$$


A simplicial complex is shellable if it has a shelling.

The existence of a shelling tells us a great deal about the topology of a pure $d$ dimensional complex: the complex is Cohen-Macaulay, with homotopy type a bouquet of spheres of dimension $d$. A fact about shellable complexes that will be especially useful for us is that a shellable proper pure $d$-dimensional subcomplex of a simplicial $d$-sphere is a $d$-ball [7, Proposition 1.2].

A cover relation in a poset $P$, denoted $x \lessdot y$, is a pair $x \leq y$ of elements in $P$ such that there is no $z \in P$ with $x \lessgtr z \lessgtr y$. Equivalently, a cover relation is an edge in the Hasse diagram of $P$.

An EL-labeling of $P$ (where $E L$ stands for edge lexicographic) is a map from the cover relations of $P$ to some fixed partially ordered set, such that in any interval $[x, y]$ there is a unique increasing maximal chain (i.e., a unique chain with increasing labels, read from the bottom), and this chain is lexicographically first among maximal chains in $[x, y]$. It is a well-known theorem of Björner in the pure case [1, Theorem 2.3], and more generally of Björner and Wachs [3, Theorem 5.8], that any bounded poset $P$ with an EL-labeling is shellable. As a result, the term EL-shelling is sometimes used as a synonym of $E L$-labeling.

The families of posets that we study in this paper will have lower intervals $[\hat{0}, x]$ that 'look like' the whole poset, but upper intervals $[x, \hat{1}]$ of a different form. For induction, then, it will usually be easier for us to label the posets upside down, and construct dual $E L$-labelings, that is, EL-labelings of the dual poset. Dual EL-labelings have been used in other settings, and are natural in many contexts [2, Corollary 4.4] [24, Corollary 4.10].

A generalization of an EL-labeling which is sometimes easier to construct (though harder to think about) is that of a $C L$-labeling. Here, instead of labeling the cover relations (edges), we label "rooted edges." More precisely, a rooted edge, or rooted cover relation, is a pair $(\mathbf{r}, x \lessdot y)$, where the root $\mathbf{r}$ is any maximal chain from $\hat{0}$ to $x$. Also, if $x_{0} \lessdot x_{1} \lessdot \cdots \lessdot x_{n}$ is a maximal chain on $\left[x_{0}, x_{n}\right]$, and $\mathbf{r}$ is a root for $x_{0} \lessdot x_{1}$, then $\mathbf{r} \cup\left\{x_{1}\right\}$ is a root for $x_{1} \lessdot x_{2}$, and so on, so it makes sense to talk of a rooted chain $\mathbf{c}_{\mathbf{r}}$

on a rooted interval $\left[x_{0}, x_{n}\right]_{\mathbf{r}}$. A $C L$-labeling is one where every rooted interval $[x, z]_{\mathbf{r}}$ has a unique increasing maximal chain, and the increasing chain is lexicographically first among all chains in $[x, z]_{\mathbf{r}}$. An in-depth discussion of $C L$-labelings can be found in [2,3]: the main fact is that $E L$-shellable $\Longrightarrow C L$-shellable $\Longrightarrow$ shellable. We will make real use of the greater generality of $C L$-labelings only in Section 5 , and the unfamiliar reader is encouraged to read " $E L$ " for " $C L$ " everywhere else.

The homotopy type of bounded posets with a $C L$-labeling (including an $E L$-labeling) is especially easy to understand, as discussed in [3]. Such a poset is homotopy equivalent to a bouquet of spheres, with the spheres in one-to-one correspondence with the descending maximal chains. These descending chains moreover form a cohomology basis for $|P|$.

\subsection{Supersolvable lattices}

The upper intervals $[x, \hat{1}]$ in the posets we look at will be supersolvable, so we mention some facts about supersolvable lattices. For additional background, the reader is referred 
to [26] or [15].

An element $x$ of a lattice $L$ is left modular if for every $y \leq z$ in $L$ it holds that $(y \vee x) \wedge z=y \vee(x \wedge z)$. This looks a great deal like the well-known Dedekind identity from group theory, and in particular any normal subgroup is left modular in the subgroup lattice.

A graded lattice is supersolvable if there is a maximal chain $\hat{1}=x_{0} \gg x_{1} \gg \cdots \gg x_{d}=\hat{0}$, where each $x_{i}$ is left modular. Thus the subgroup lattice of a supersolvable group is a supersolvable lattice. In fact, supersolvable lattices were introduced to generalize the lattice properties of supersolvable groups.

A supersolvable lattice has a dual EL-labeling

$$
\lambda^{s s}(y>z)=\min \left\{j: x_{j} \wedge y \leq z\right\}=\max \left\{j-1: x_{j} \vee z \geq y\right\},
$$

which we call the supersolvable labeling of $L$ (relative to the given chain of left modular elements). This labeling has the property:

Given an interval $[x, y]$, every chain on $[x, y]$ has the same set of labels (in different orders).

McNamara [14] has shown that having an EL-labeling that satisfies (2) characterizes the supersolvable lattices.

\subsection{Cohen-Macaulay complexes}

If $F$ is a face in a simplicial complex $\Delta$, then the link of $F$ in $\Delta$ is

$$
\operatorname{link}_{\Delta} F=\{G \in \Delta: G \cap F=\emptyset \text { and } G \cup F \in \Delta\} .
$$

A simplicial complex $\Delta$ is Cohen-Macaulay if the link of every face has the homology of a bouquet of top dimensional spheres, that is, if $\tilde{H}_{i}\left(\operatorname{link}_{\Delta} F\right)=0$ for all $i<\operatorname{dim}\left(\operatorname{link}_{\Delta} F\right)$.

The Cohen-Macaulay property has a particularly nice formulation on the order complex of a poset. A poset is Cohen-Macaulay if every interval $[x, y]$ has $\tilde{H}_{i}([x, y])=0$ for all $i<\operatorname{dim}(|[x, y]|)$. In particular, every interval in a Cohen-Macaulay poset is CohenMacaulay. It is well-known that every shellable complex is Cohen-Macaulay. For a proof of this fact and additional background on Cohen-Macaulay complexes and posets, see [28].

The Cohen-Macaulay property is essentially a connectivity property. Just as we say a graph $G$ is doubly connected (or 2-connected) if $G$ is connected and $G \backslash\{v\}$ is connected for each $v \in G$, we say that a simplicial complex $\Delta$ is doubly Cohen-Macaulay (2-CM) if

1. $\Delta$ is Cohen-Macaulay, and

2. for each vertex $x \in \Delta$, the induced complex $\Delta \backslash\{x\}$ is Cohen-Macaulay of the same dimension as $\Delta$.

Doubly Cohen-Macaulay complexes are closely related to complexes with convex ear decompositions: 
Theorem 2.3. (Swartz [31]) If $\Delta$ has a convex ear decomposition, then $\Delta$ is doubly Cohen-Macaulay.

Thus, convex ear decompositions can be thought of as occupying an analogous role to shellings in the geometry of simplicial complexes: a shelling is a combinatorial reason for a complex to be (homotopy) Cohen-Macaulay, and a convex ear decomposition is a combinatorial reason for a complex to be doubly Cohen-Macaulay. Of course, convex ear decompositions also give the strong enumerative constraints of Theorems 2.1 and 2.2.

Intervals in a poset with a convex ear decomposition are not known to have convex ear decompositions. However, intervals do inherit the 2-CM property, as intervals are links in the order complex, and intervals inherit the Cohen-Macalay property. Thus, Theorem 2.3 is particularly useful in proving that a poset does not have a convex ear decomposition.

\section{$2.5 \quad E L$-ceds and $C L$-ceds}

Nyman and Swartz used an EL-labeling in [17] to find a convex ear decomposition for any geometric lattice. The condition on an EL-labeling says that ascending chains are unique in every interval, and that the lexicographic order of maximal chains is a shelling. Starting with the usual EL-labeling of a geometric lattice, Nyman and Swartz showed that descending chains are unique in intervals of an ear of their decomposition, and that the reverse of the lexicographic order is a shelling. Schweig used similar techniques in [23] to find convex ear decompositions for several families of posets, including supersolvable lattices with complemented intervals.

In this subsection, we axiomatize the conditions necessary for these techniques. Although we state everything in terms of $C L$-labelings, one could just as easily read ' $E L$ ' for the purposes of this section, and ignore the word 'rooted' whenever it occurs.

Suppose that $P$ is a bounded poset of rank $k$. Let $\left\{\Sigma_{s}\right\}$ be an ordered collection of rank $k$ subposets of $P$. For each $s$, let $\Delta_{s}$ be the simplicial subcomplex generated by all maximal chains that occur in $\Sigma_{s}$, but not in any $\Sigma_{t}$ for $t<s$. (Informally, $\Delta_{s}$ is all "new" maximal chains in $\Sigma_{s}$.) Recall that $\mathcal{M}\left(\Sigma_{s}\right)$ refers to the maximal chains of $\Sigma_{s}$, and let $\mathcal{M}\left(\Delta_{s}\right)$ be the maximal chains of $\Delta_{s}$. As usual, maximal chains are in bijective correspondence with facets of the order complex via removing or adding $\hat{1}$ and $\hat{0}$.

The ordered collection $\left\{\Sigma_{s}\right\}$ is a chain lexicographic convex ear decomposition (or $C L$ ced for short) of $P$ with respect to the $C L$-labeling $\lambda$, if it obeys the following properties:

CLced-polytope For each $s, \Sigma_{s}$ is the face lattice of a convex polytope.

CLced-desc For any $\Delta_{s}$ and rooted interval $[x, y]_{\mathbf{r}}$ in $P$, there is at most one descending maximal chain $\mathbf{c}$ on $[x, y]_{\mathrm{r}}$ which is a face of $\Delta_{s}$.

CLced-bdry If $\mathbf{c}$ is a chain of length $<k$, such that $\mathbf{c}$ can be extended to a maximal chain in both of $\Delta_{s}$ and $\Delta_{t}$, where $t<s$; then $\mathbf{c}$ can be extended to a chain in $\mathcal{M}\left(\Sigma_{s}\right) \backslash \mathcal{M}\left(\Delta_{s}\right)$.

CLced-union Every chain in $P$ is in some $\Sigma_{s}$. 
Note 2.4. We note the resemblance of (CLced-desc) with the increasing chain condition for a $C L$-labeling (under the reverse ordering of labels); but though $\Delta_{s}$ is a simplicial complex corresponding with chains in $P$, it is not itself a poset.

Note 2.5. By analogy with $C L$-labelings, it would seem that we should require the descending chain in (CLced-desc) to be lexicographically last. But this would be redundant: suppose $\mathbf{c}$ is the lexicographically last maximal chain in $[x, y]_{\mathbf{r}}$ that is also in $\Delta_{s}$, but that c has an ascent at $c_{i}$. Then Lemma 2.7 below gives that we can replace the ascent with a descent, obtaining a lexicographically later chain, a contradiction.

Note 2.6. As previously mentioned, we will usually refer to $E L$-ceds in this paper, i.e., the special case where $\lambda$ is an $E L$-labeling. Similarly, we may refer to dual $E L$-ceds, that is, $E L$-ceds of the dual poset.

Lemma 2.7. (Technical Lemma) Let $\left\{\Sigma_{s}\right\}$ be a $C L$-ced of a poset, with $\left\{\Delta_{s}\right\}$ as above, and let $\mathbf{c}=\left\{x \lessdot c_{1} \lessdot \cdots \lessdot c_{j-1} \lessdot y\right\}$ be a maximal chain on a rooted interval $[x, y]_{\mathbf{r}}$, with $\mathbf{c}$ a face in $\Delta_{s}$. Suppose that $\mathbf{c}$ has an ascent at $c_{i}$. Then $\Delta_{s}$ contains a $\mathbf{c}^{\prime \prime}=\left(\mathbf{c} \backslash\left\{c_{i}\right\}\right) \cup c_{i}^{\prime \prime}$ which descends at $c_{i}^{\prime \prime}$, and is lexicographically later than $\mathbf{c}$.

Proof. Let $\mathbf{c}^{-}=\mathbf{c} \backslash\left\{c_{i}\right\}$, and let $\Sigma_{t}$ be the first subposet in the $C L$-ced that contains $\mathbf{c}^{-}$. Since $\Sigma_{t}$ is the face lattice of a polytope, it is Eulerian, so $\mathbf{c}^{-}$has two extensions in $\Sigma_{t}$. By the uniqueness of ascending chains in $C L$-labelings, at most one is ascending at rank $i$; by (CLced-desc), at most one is descending. Thus, there is exactly one of each. The extension with the ascent is $\mathbf{c}$, call the other extension $\mathbf{c}^{\prime \prime}$.

We have shown that $\mathbf{c}$ is in $\Sigma_{t}$ and (since $\Sigma_{t}$ is the first subposet containing $\mathbf{c}^{-}$) that $s=t$, so that $\mathbf{c}^{\prime \prime}$ is in $\Delta_{s}$. Finally, $\mathbf{c}^{\prime \prime}$ is lexicographically later than $\mathbf{c}$ by the definition of $C L$-labeling.

We also recall a useful lemma from undergraduate point-set topology [16, Exercise 17.19]:

Lemma 2.8. If $B$ is a closed subset of $X$, then $\partial B=B \cap \overline{X \backslash B}$.

Although they did not use the terms " $C L$-ced" or " $E L$-ced" in their paper, the essence of the following theorem was proved by Nyman and Swartz in [17, Section 4], where they used it to construct convex ear decompositions of geometric lattices.

Theorem 2.9. If $\left\{\Sigma_{s}\right\}$ is an $C L$-ced for $P$, then the associated subcomplexes $\left\{\Delta_{s}\right\}$ form a convex ear decomposition for $|P|$.

Proof. (Nyman and Swartz [17, Section 4]) The property (ced-union) follows directly from (CLced-union), and (ced-polytope) follows from (CLced-polytope) because the barycentric subdivision of a polytope is again a polytope.

For (ced-bdry), we first note that $\partial \Delta_{s}=\partial\left(\overline{\left|\Sigma_{s}\right| \backslash \Delta_{s}}\right)$ (the topological closure), hence $\partial \Delta_{s} \subseteq \Delta_{s} \cap\left(\bigcup_{t<s} \Delta_{t}\right)$. Conversely, if $\mathbf{c}$ is in $\Delta_{s} \cap\left(\bigcup_{t<s} \Delta_{t}\right)$, then (CLced-bdry) gives that c is in both $\Delta_{s}$ and $\overline{\left|\Sigma_{s}\right| \backslash \Delta_{s}}$. Lemma 2.8 then gives the desired inclusion. 
It remains to check (ced-topology). Using (CLced-desc), we show that the reverse of the lexicographic order is a shelling of $\Delta_{s}$. For if

$$
\begin{aligned}
& \mathbf{c}=\left\{\hat{0} \lessdot c_{1} \lessdot \cdots \lessdot c_{k-1} \lessdot \hat{1}\right\} \quad \text { and } \\
& \mathbf{c}^{\prime}=\left\{\hat{0} \lessdot c_{1}^{\prime} \lessdot \cdots \lessdot c_{k-1}^{\prime} \lessdot \hat{1}\right\}
\end{aligned}
$$

are maximal chains in $\Delta_{s}$, with $\mathbf{c}$ lexicographically earlier than $\mathbf{c}^{\prime}$, then (CLced-desc) and Note 2.5 give that $\mathbf{c}$ has an ascent on some interval where $\mathbf{c}$ disagrees with $\mathbf{c}^{\prime}$. So $\mathbf{c}$ has an ascent at $i$, and $c_{i} \neq c_{i}^{\prime}$.

Apply Lemma 2.7 on the interval $[\hat{0}, \hat{1}]$ to get $\mathbf{c}^{\prime \prime}$ in $\Delta_{s}$ which descends at $i$, and otherwise is the same as $\mathbf{c}$. Then $\mathbf{c}^{\prime} \cap \mathbf{c} \subseteq \mathbf{c}^{\prime \prime} \cap \mathbf{c}=\mathbf{c} \backslash\left\{c_{i}\right\}$, so $\left|\mathbf{c}^{\prime \prime} \cap \mathbf{c}\right|=|\mathbf{c}|-1$, and so $\mathbf{c}^{\prime \prime}$ is lexicographically later than $\mathbf{c}$, as Condition (1) requires for a shelling.

We now check that $\Delta_{s}$ is a proper subcomplex of $\left|\Sigma_{s}\right|$ for $s \geq 2$. Suppose that $\Delta_{s}=\left|\Sigma_{s}\right|$. Then by Notes 2.4 and $2.5, \lambda$ is a $C L$-labeling on $\Sigma_{s}$ with respect to the reverse ordering of its label set. Since $\left|\Sigma_{s}\right|$ is a sphere, there is an ascending chain (descending chain with respect to the reverse ordering) in $\Sigma_{s}$. Since the ascending chain in $P$ is unique, we have $s=1$.

By definition $\Delta_{1}=\left|\Sigma_{1}\right|$ is a $(k-2)$-sphere. Now since $\Delta_{s}$ is shellable and a proper subcomplex of the $(k-2)$-sphere $\left|\Sigma_{s}\right|$ for $s \geq 2$, we get that $\Delta_{s}$ is a $(k-2)$-ball; thus (ced-topology) holds.

Note 2.10. Each non-empty ear of $\left\{\Delta_{s}\right\}$ contains exactly one descending chain. This is no accident: see the discussion at the end of Section 2.2 .

Corollary 2.11. The following families of posets have EL-ceds, thus convex ear decompositions.

1. (Nyman and Swartz [17, Section 4]) Geometric lattices.

2. (Schweig [23, Theorem 3.2]) Supersolvable lattices with Möbius function non-zero on every interval.

3. (Schweig [23, Theorems 5.1 and 7.1]) Rank-selected subposets of supersolvable and geometric lattices.

In the following two sections, we will exhibit an $E L$-ced for the $d$-divisible partition lattice, and (using only slightly different techniques) a convex ear decomposition for the coset lattice of a relatively complemented group.

\section{The $d$-divisible partition lattice}

The $d$-divisible partition poset, denoted $\bar{\Pi}_{n}^{d}$, is the set of all proper partitions of $[n]=$ $\{1, \ldots, n\}$ where each block has cardinality divisible by $d$. The $d$-divisible partition lattice, denoted $\Pi_{n}^{d}$ is $\bar{\Pi}_{n}^{d}$ with a 'top' $\hat{1}$ and 'bottom' $\hat{0}$ adjoined. $\Pi_{n}^{d}$ is ordered by refinement 
(which we denote by $\prec$ ), as in the usual partition lattice $\Pi_{n}\left(=\Pi_{n}^{1}\right)$. In general, $\Pi_{n}^{d}$ is a subposet of $\Pi_{n}$, with equality in the case $d=1$; on the other hand, intervals $[a, \hat{1}]$ are isomorphic to $\Pi_{n / d}$ for any atom $a \in \Pi_{n}^{d}$. We refer frequently to [33] for information about the $d$-divisible partition lattice.

As $\Pi_{n}$ is a supersolvable geometric lattice, and hence quite well understood, we restrict ourself to the case $d>1$. It will sometimes be convenient to partition a different set $S \neq[n]$. In this case we write $\Pi_{S}$ to be the set of all partitions of $S$, and $\Pi_{S}^{d}$ the set of all $d$-divisible partitions of $S$, so that $\Pi_{n}^{d}=\Pi_{[n]}^{d}$ is a special case.

Wachs found a homology basis for $\Pi_{n}^{d}$ in $[33$, Section 2]. We recall her construction. By $S_{n}$ we denote the symmetric group on $n$ letters. We will write a permutation $\alpha \in S_{n}$ as a word $\alpha(1) \alpha(2) \ldots \alpha(n)$, and define the descent set of $\alpha$ to be the indices where $\alpha$ descends, i.e., $\operatorname{des} \alpha=\{i \in[n-1]: \alpha(i)>\alpha(i+1)\}$.

Then a split of $\alpha \in S_{n}$ at di divides $\alpha$ into $\alpha(1) \alpha(2) \ldots \alpha(d i)$ and $\alpha(d i+1) \ldots \alpha(n)$.

A switch-and-split at position $d i$ does the same, but first transposes ('switches') $\alpha(d i)$ and $\alpha(d i+1)$.

These operations can be repeated, and the result of repeated applications of splits and switch-and-splits at $d$-divisible positions is a $d$-divisible partition. For example, if $\alpha=561234$, then the 2-divisible partition 56|13|24 results from splitting at position 2 and switch-and-splitting at position 4 .

Let $\Sigma_{\alpha}$ be the subposet of $\Pi_{n}^{d}$ that consists of all partitions that are obtained by splitting and/or switch-and-splitting the permutation $\alpha$ at positions divisible by $d$. Let

$$
A_{n}^{d}=\left\{\alpha \in S_{n}: \alpha(n)=n, \operatorname{des} \alpha=\{d, 2 d, \ldots, n-d\}\right\} .
$$

Wachs proved

Theorem 3.1. (Wachs [33, Theorems 2.1-2.2])

1. $\Sigma_{\alpha}$ is isomorphic to the face lattice of the $\left(\frac{n}{d}-1\right)$-cube for any $\alpha \in S_{n}$.

2. $\left\{\Sigma_{\alpha}: \alpha \in A_{n}^{d}\right\}$ is a basis for $H_{*}\left(\Pi_{n}^{d}\right)$.

After some work, this basis will prove to be a dual $E L$-ced.

\subsection{A dual $E L$-labeling for $\Pi_{n}^{d}$}

In addition to the homology basis already mentioned, Wachs constructs an $E L$-labeling in [33, Section 5], by taking something close to the standard $E L$-labeling of the geometric lattice on intervals $[a, \hat{1}] \cong \Pi_{n / d}$ (for $a$ an atom), and "twisting" by making selected labels negative. While her labeling is not convenient for our purposes, we use her sign idea to construct our own dual $E L$-labeling starting with a supersolvable $E L$-labeling of $[a, \hat{1}]$.

Partition lattices were one of the first examples of supersolvable lattices to be studied [26]. It is not difficult to see that the maximal chain with $j$ th ranked element

$$
1|2| \ldots|j|(j+1) \ldots n
$$


is a left modular chain in $\Pi_{n}$.

Let $y \succ z$ be a cover relation in $\Pi_{n}$. Then $y$ is obtained by merging two blocks $B_{1}$ and $B_{2}$ of the partition $z$, where without loss of generality $\max B_{1}<\max B_{2}$. The supersolvable dual $E L$-labeling (relative to the above chain of left modular elements) is especially natural:

$$
\begin{aligned}
\lambda^{s s}(y \succ z) & =\min \{j:(1|\ldots| j \mid(j+1) \ldots n) \wedge y \prec z\} \\
& =\max B_{1} .
\end{aligned}
$$

We now construct the labeling that we will use for $\Pi_{n}^{d}$. Let $y \succ z$ be a cover relation in $\Pi_{n}^{d}$, where $z \neq \hat{0}$. As above, $y$ is obtained by merging blocks $B_{1}$ and $B_{2}$ of $z$, where $\max B_{1}<\max B_{2}$. Label

$$
\begin{aligned}
& \lambda(y \succ z)=\left\{\begin{aligned}
-\max B_{1} & \text { if } \max B_{1}<\min B_{2}, \\
\max B_{1} & \text { otherwise, and }
\end{aligned}\right. \\
& \lambda(y \succ \hat{0})=0 .
\end{aligned}
$$

When discussing dual EL-labelings, any reference to ascending or descending chains is in the dual poset, so that the inequalities go in the opposite direction from normal.

Note 3.2. Let $a \in \Pi_{n}^{d}$ be an atom. Then $a$ has $n / d$ blocks, and every block has $d$ elements. Order the blocks $\left\{B_{i}\right\}$ so that $\max B_{1}<\max B_{2}<\cdots<\max B_{n / d}$, and let $\mathcal{B}=\left\{\max B_{1}, \ldots, \max B_{n / d}\right\}$. Then $[a, 1] \cong \Pi_{\mathcal{B}}$, and we recognize $|\lambda|$ as the supersolvable dual $E L$-labeling $\lambda^{s s}$ on $\Pi_{\mathcal{B}}$.

Note 3.3. We also can view $\Pi_{n}^{d}$ as a subposet of $\Pi_{n}$. A cover relation $y \succ z$ in $\Pi_{n}^{d}$ is a cover relation in $\Pi_{n}$ unless $z=\hat{0}$. Thus, $|\lambda|$ is the restriction of $\lambda^{s s}$ on $\Pi_{n}$, except at the bottom edges $y \succ \hat{0}$.

Note 3.4. The cover relation $x_{0} \succ x_{1}$ gets a negative label if and only if $B_{1} \mid B_{2}$ is a noncrossing partition of $B=B_{1} \cup B_{2}$. We will call this a non-crossing refinement of $x_{0}$. The poset of all non-crossing partitions has been studied extensively [25, 13], although this seems to have a different flavor from what we are doing. Also related is the connectivity set of a permutation [29], the set of positions at which a split yields a non-crossing partition.

Recall that if $P_{1}$ and $P_{2}$ are posets, then their direct product $P_{1} \times P_{2}$ is the Cartesian product with the ordering $\left(x_{1}, x_{2}\right) \leq\left(y_{1}, y_{2}\right)$ if $x_{1} \leq x_{2}$ and $y_{1} \leq y_{2}$. The lower reduced product $P_{1} \check{\times} P_{2}$ of two bounded posets is $\left(\left(P_{1} \backslash\{\hat{0}\}\right) \times\left(P_{2} \backslash\{\hat{0}\}\right)\right) \cup\{\hat{0}\}$. Although the definition of the lower reduced product may appear strange at first glance, it occurs naturally in many settings, including the following easily-proved lemma:

Lemma 3.5. Let $y \succ x$ be elements of $\Pi_{n}^{d} \backslash\{\hat{0}\}$, with $y=B_{1}|\ldots| B_{k}$. Then

1. $[\hat{0}, y] \cong \Pi_{B_{1}}^{d} \check{\times} \Pi_{B_{2}}^{d} \check{\times} \ldots \check{\times} \Pi_{B_{k}}^{d}$.

2. $[y, \hat{1}] \cong \Pi_{k}$.

3. $[x, y]$ is the direct product of intervals in $\Pi_{B_{i}}^{d}$. 
Note 3.6. We discuss (lower/upper reduced) products of posets at much more length in Section 5. Although the situation with $\Pi_{n}^{d}$ is simple enough that we do not need to refer directly to product labelings (introduced in Section 5.3), they are the underlying reason we can look at partitions block by block in the proofs that follow.

Theorem 3.7. $\lambda$ is a dual EL-labeling of $\Pi_{n}^{d}$.

Proof. We need to show that each interval has a unique (dual) increasing maximal chain which is lexicographically first. There are two forms of intervals we must check:

Case 1. Intervals of the form $\left[\hat{0}, x_{0}\right]$.

Since the bottommost label on every chain in $\left[\hat{0}, x_{0}\right]$ is a 0 , every other label in an increasing chain must be negative. Hence, every edge $x_{i} \succ x_{i+1}$ in an increasing chain must correspond to a non-crossing refinement of $x_{i}$.

In such a chain, any block $B$ of $x_{0}$ is partitioned repeatedly into non-crossing sub-blocks. At the atom level, this block $B$ is sub-partitioned as $B_{1}|\ldots| B_{k}$, where $\max B_{i}<$ $\min B_{i+1}$. Thus, any increasing chain on $\left[\hat{0}, x_{0}\right]$ passes through this single atom, and we have reduced the problem to Case 2 .

Case 2. Intervals of the form $\left[x_{m}, x_{0}\right]$, where $x_{m} \neq \hat{0}$.

By Lemma 3.5 and the discussion following, it suffices to examine a single block $B$ of $x_{0}$. (The labels on disjoint blocks are independent of each other.)

In $x_{m}$, let $B$ be subpartitioned as $B_{1}|\ldots| B_{k}$, with $\max B_{s}=b_{s}$ and $b_{1}<b_{2}<\cdots<b_{k}$. The edges we consider correspond with subpartitioning $B$ between itself and $B_{1}|\ldots| B_{k}$.

First, we show that the lexicographically first chain $\mathbf{c}=x_{0} \succ x_{1} \succ \ldots \succ x_{m}$ is unique. If there are any negative labels down from $x_{i}$, the edge $x_{i} \succ x_{i+1}$ will have the label $-b_{s}$ with greatest absolute value among negative labels. Thus,

$$
x_{i+1}=x_{i} \wedge\left(B_{1} \ldots B_{s} \mid B_{s+1} \ldots B_{k}\right)
$$

and hence $x_{i} \succ x_{i+1}$ is the unique edge down from $x_{i}$ with this label. Otherwise, $x_{i} \succ x_{i+1}$ will have the least possible (positive) label, which is unique since $|\lambda|$ is a dual supersolvable EL-labeling on $\left[x_{m}, x_{0}\right]$.

Next, we show that the lexicographically first chain is increasing. Suppose that $\mathbf{c}$ has a descent at $x_{i-1} \succ x_{i} \succ x_{i+1}$, with $\lambda\left(x_{i-1} \succ x_{i}\right)=\alpha$ and $\lambda\left(x_{i} \succ x_{i+1}\right)=\beta$, corresponding to dividing a block $C$ as

$$
C \succ C_{1}\left|C_{2} \cup C_{3} \succ C_{1}\right| C_{2} \mid C_{3}
$$

Since $|\lambda|$ is a dual $E L$-labeling, both labels cannot be positive. Thus, $\beta<0$. If then $|\alpha|<|\beta|$, we have $\max C_{1}<\max C_{2}<\min C_{3}$, and then $C \succ C_{1} \cup C_{2} \mid C_{3}$ is noncrossing, with a $\beta$ label, and so lexicographically before $x_{i-1} \succ x_{i}$. Otherwise, $|\alpha|>|\beta|$. Since we have a descent at $i$, we see $\alpha>0$, and so the $\pm \beta<\alpha$ label on the edge obtained by partitioning $C \succ C_{1} \cup C_{2} \mid C_{3}$ is again lexicographically before $x_{i-1} \succ x_{i}$. In either case, we have shown that any $\mathbf{c}$ with a descent is not lexicographically first. 
Finally, we show that any increasing chain is lexicographically first. Suppose that there is an edge $x_{0} \succ y\left(\succ x_{m}\right)$ that receives a $-b_{s}$ label. Then $y=B_{1} \ldots B_{s} \mid B_{s+1} \ldots B_{k}$ is a non-crossing partition of $B$, and in particular $B_{s}<B_{s+1}, \ldots, B_{k}$. We see that any subpartion of $x_{0}$ separating $B_{s}$ from $B_{t}$ for $t>s$ is non-crossing, thus every chain on $\left[x_{m}, x_{0}\right]$ has a $-b_{s}$ label. This fact, combined with (2) shows that any increasing chain on an interval must be constructed inductively by repeatedly taking the least-labeled edge down, hence be lexicographically first.

The descending chains of Wachs's EL-labeling are $\left\{r_{\sigma}: \sigma \in A_{n}^{d}\right\}$, where $r_{\sigma}$ corresponds to successively splitting $\sigma$ at the greatest possible $\sigma(i d)$ [33, Theorem 5.2]. It is easy to see that each $r_{\sigma}$ is also descending with respect to our dual $E L$-labeling, and a dimension argument shows us that $\left\{r_{\sigma}: \sigma \in A_{n}^{d}\right\}$ is exactly the set of descending chains.

\subsection{An $E L$-ced for $\Pi_{n}^{d}$}

Order $\left\{\Sigma_{\alpha}\right\}$ lexicographically by the reverse of the words $\alpha$ according to the reverse ordering on $[n]$. That is, order lexicographically by the words $\alpha(n) \alpha(n-1) \cdots \alpha(1)$, where $n \triangleleft n-1 \triangleleft \cdots \triangleleft 1$. We refer to this ordering as $r r$-lex, for "reverse reverse lexicographic." For example, 132546 is the first permutation in $A_{6}^{2}$ with respect to $r r$-lex, while $231546<_{\text {rr-lex }} 142536$ (since $4>3$ in position 5 ).

We will prove the following version of Theorem 1.1. Let $\Sigma_{\alpha}$ be as in the text preceding Theorem 3.1, and $\lambda$ as in Section 3.1.

Theorem 3.8. $\left\{\Sigma_{\alpha}: \alpha \in A_{n}^{d}\right\}$ ordered by $r r$-lex is a dual EL-ced of $\Pi_{n}^{d}$ with respect to $\lambda$.

We introduce some terms. If $B_{1}|\ldots| B_{k}$ is a partition of $[n]$, then we say that $\alpha \in S_{n}$ has the form $B_{1} B_{2} \ldots B_{k}$ if the first $\left|B_{1}\right|$ elements in the word $\alpha$ are in $B_{1}$, the next $\left|B_{2}\right|$ are in $B_{2}$, and so forth. When $k=2$, we say that $\alpha$ has switched form $B_{1} B_{2}$ if $\alpha^{\prime}$ has the form $B_{1} B_{2}$ for $\alpha^{\prime}=\alpha \circ\left(\left|B_{1}\right|\left|B_{1}\right|+1\right)$, that is, for $\alpha^{\prime}$ equal to $\alpha$ composed with the transposition of adjacent elements at $\left|B_{1}\right|$.

We can also talk of $\alpha$ having form $B_{1} B_{2} \ldots B_{k}$ up to switching, by which we mean some $\alpha^{\prime}$ has the form $B_{1} \ldots B_{k}$, where $\alpha^{\prime}$ is $\alpha$ up to transpositions at the borders of some (but not necessarily all) of the blocks. Finally, if $B \subseteq[n]$, then $\left.\alpha\right|_{B}$ is the word $\alpha=\alpha(1) \alpha(2) \ldots \alpha(n)$ with all $\alpha(i)$ 's that are not in $B$ removed.

Example 3.9. If $B_{1}=\{1,2,3\}$ and $B_{2}=\{4,5,6\}$, then 123456,321654 , and 213465 all have the form $B_{1} B_{2}$. 124356 and 135246 have switched form $B_{1} B_{2}$, while 152346 does not have the form $B_{1} B_{2}$, even up to switching.

Clearly, the $d$-divisible partition $B_{1}|\ldots| B_{k}$ is in $\Sigma_{\alpha}$ if and only if $\alpha$ has the form $B_{1} B_{2} \ldots B_{k}$ up to switching.

Lemma 3.10. Every maximal chain $\mathbf{c}$ in $\Pi_{n}^{d}$ is in $\Sigma_{\alpha}$ for some $\alpha \in A_{n}^{d}$. 
Proof. We will in fact construct the earliest such $\alpha$ according to the rr-lex ordering, which will in turn help us with Corollary 3.11. The proof has a similar feel to the well-known quicksort algorithm. Let $\mathbf{c}=\left\{\hat{1}=c_{0} \succ \ldots \succ c_{n / d}=\hat{0}\right\}$.

Consider first the edge $\hat{1} \succ c_{1}$ in c. The edge splits $[n]$ into $B_{1} \mid B_{2}$, and clearly such $\alpha$, if it exists, must have the form $B_{1} B_{2}$ or $B_{2} B_{1}$ up to switching. If $\max B_{1}<\max B_{2}$, then all permutations in $A_{n}^{d}$ of the (possibly switched) form $B_{1} B_{2}$ come before permutations of the (possibly switched) form $B_{2} B_{1}$, so the rr-lex first $\alpha$ with $\mathbf{c}$ in $\Sigma_{\alpha}$ has the form $B_{1} B_{2}$ up to switching.

Apply this argument inductively down the chain. At $c_{i}$, we will have shown that the rr-lex first $\alpha$ with $\mathbf{c}$ in $\Sigma_{\alpha}$ must have the form $B_{1} B_{2} \cdots B_{i+1}$ up to switching. Then if $c_{i} \succ c_{i+1}$ splits block $B_{j}$ into $B_{j, 1}$ and $B_{j, 2}$, with $\max B_{j, 1}<\max B_{j, 2}$, an argument similar to that with $\hat{1} \succ c_{1}$ gives that $\alpha$ must in fact have the form

$$
B_{1} B_{2} \ldots B_{j-1} B_{j, 1} B_{j, 2} B_{j+1} \ldots B_{i+1}
$$

up to switching.

At the end, we have shown the earliest $\alpha$ having $\mathbf{c}$ in $\Sigma_{\alpha}$ must have the form $B_{1} \ldots B_{n / d}$ up to switching. Conversely, it is clear from the above that for any $\alpha$ of this form, $\mathbf{c}$ is in $\Sigma_{\alpha}$. Sort the elements of each $B_{i}$ in ascending order to get a permutation $\alpha_{0}$. This $\alpha_{0}$ is in $S_{n}$ but not necessarily in $A_{n}^{d}$, so we perform a switch at each $d$-divisible position where there is an ascent (i.e., where $B_{i}<B_{i+1}$ ). This gives us an element $\alpha \in A_{n}^{d}$ of the given form up to switching, and finishes the proof of the statement.

We continue nonetheless to finish showing that $\alpha$ is the first element in $A_{n}^{d}$ with c in $\Sigma_{\alpha}$. We need to show that if $\beta$ is another element of $A_{n}^{d}$ with the same form up to switching of $B_{1} B_{2} \ldots B_{n / d}$ (but different switches), then $\beta>_{\text {rr-lex }} \alpha$. If $B_{i}<B_{i+1}$, then both $\alpha$ and $\beta$ are switched at $i d$ (as otherwise we are not in $A_{n}^{d}$ ). Otherwise, if $\beta$ is a switch at $i d$, then the switch exchanges $\beta(i d)$ and $\beta(i d+1)$ (up to resorting the blocks). Since $\beta(i d)>\beta(i d+1)$, "unswitching" moves a larger element of $[n]$ later in the permutation, yielding an rr-lex earlier element of the given form up to switching.

Let $\Delta_{\alpha}$ be the simplicial complex generated by maximal chains that are in $\Sigma_{\alpha}\left(\alpha \in A_{n}^{d}\right)$, but in no $\Sigma_{\beta}$ for $\beta \in A_{n}^{d}$ with $\beta<_{\text {rr-lex }} \alpha$. In the following corollary, we summarize the information from the proof of Lemma 3.10 about the form of $\alpha$ with $\mathbf{c}$ in $\Delta_{\alpha}$.

Corollary 3.11. Let $\mathbf{c}$ be a maximal chain in $\Delta_{\alpha}$, with $y \cdot x$ an edge in $\mathbf{c}$ which merges blocks $B_{1}$ and $B_{2}$ into block $B\left(\max B_{1}<\max B_{2}\right)$. Then

1. $\alpha$ has the form ... $B_{1} B_{2} \ldots$, up to switching.

2. Let $\tau_{i d}$ be the transposition exchanging $i d$ and $i d+1$. If $y \succ x$ corresponds to a switch-and-split at $i d$, then the permutation $\alpha \circ \tau_{i d}$ is ascending between positions $(i-1) d+1$ and $(i+1) d$.

3. $\left.\alpha\right|_{B_{1}}=\ldots \max B_{1}$, i.e., $\max B_{1}$ is rightmost in $\left.\alpha\right|_{B_{1}}$. 
Proof. (1) and (2) are clear from the proof of Lemma 3.10.

For (3), suppose that $\max B_{1}$ is not rightmost in $\left.\alpha\right|_{B_{1}}$. Then since $\alpha$ is ascending on $d$-segments, we have that $\max B_{1}$ is rightmost in some $d$-segment of $\left.\alpha\right|_{B_{1}}$. If a switchand-split occurs at $\max B_{1}$ then we have a contradiction of (2), while a split contradicts (1).

Every chain passing through an atom $a$ has the same labels up to sign, and Corollary 3.11 tells us what the labels are. It is now not difficult to prove (CLced-desc) and (CLcedbdry).

Proposition 3.12. Let $\left[x_{m}, x_{0}\right]$ be an interval with $x_{m}, x_{0} \in \Delta_{\alpha}$. Then there is at most one (dual) descending maximal chain $\mathbf{c}$ on $\left[x_{m}, x_{0}\right]$ which is in $\Delta_{\alpha}$.

Proof. There are two cases:

Case 1. $x_{m}=\hat{0}$.

It suffices to consider a block $B$ of $x_{0}$. Partitions of $B$ corresponding to edges in $\Sigma_{\alpha}$ must either split or switch-and-split $\left.\alpha\right|_{B}$ at $d$-divisible positions, and as every chain on $\left[\hat{0}, x_{0}\right]$ has bottommost label 0, all other edges of a descending chain must have positive labels (and so correspond to crossing partitions).

Claim 3.13. All edges of such a descending chain correspond to splittings of $\alpha$.

Proof. (of Claim) Suppose otherwise. Without loss of generality we can assume that $x_{0} \succ x_{1}$ in $\mathbf{c}$ corresponds to a switch-and-split of $B$ into $B_{1} \mid B_{2}$, with $B$ the block of smallest size which is switch-and-split by an edge in $\mathbf{c}$. We will show that $\mathbf{c}$ has an ascent.

Corollary 3.11 part 2 tells us that the first $d$ letters in $\left.\alpha\right|_{B_{2}}$ are strictly greater than the last $d$ in $\left.\alpha\right|_{B_{1}}$, and since max $B_{1}$ is rightmost in $\left.\alpha\right|_{B_{1}}$, that the first $d$ letters of $\left.\alpha\right|_{B_{2}}$ are strictly greater than all of $B_{1}$. Since $\lambda\left(x_{m-1} \succ \hat{0}\right)=0$, any negative label gives an ascent. If $\left|B_{2}\right|=d$, then we have shown that $B_{1} \mid B_{2}$ is non-crossing (giving a negative label). If on the other hand $\left|B_{2}\right|>d$, then any subdivision of $B_{2}$ gives a label with absolute value $>\max B_{1}$, hence an ascent. In either case, we contradict $\mathbf{c}$ being a descending chain.

It follows immediately that a descending chain on $\left[\hat{0}, x_{0}\right]$ is unique.

Case 2. $x_{m} \neq \hat{0}$.

As usual, we consider what happens to a block $B$ of $x_{0}$. In $x_{m}$, let $B$ partition as $B_{1}|\ldots| B_{k}$, where $\left.\alpha\right|_{B}$ has the form $B_{1} B_{2} \ldots B_{k}$ up to switching. Then every edge in $\Delta_{\sigma}$ comes from subdividing at some $B_{i}$, i.e., as shown at the dotted line here

$$
\cdots \cup B_{j}\left|B_{j+1} \cup \cdots \cup B_{i} \vdots B_{i+1} \cup \cdots \cup B_{l}\right| \ldots
$$

Let $b_{i}=\max B_{i}$, so every chain on $\left[x_{m}, x_{0}\right]$ has labels $\pm b_{1}, \pm b_{2}, \ldots, \pm b_{k-1}$. 
Suppose that $\cdots \cup B_{i} \mid B_{i+1} \cup \ldots$ is crossing, but $B_{i} \mid B_{i+1}$ is non-crossing. Corollary 3.11 part 3 tells us that $\max \left(\cdots \cup B_{i}\right)=\max B_{i}$, so that

$$
\min \left(B_{i+2} \cup \cdots \cup B_{k}\right)<\max B_{i}<\min B_{i+1}<\max B_{i+1} .
$$

It follows that $B_{i+1} \mid B_{i+2} \cup \ldots$ is also crossing. Thus, if $B_{i} \mid B_{i+1}$ is non-crossing, then (positive) $b_{i}$ is not the label of the first edge of a descending chain $\mathbf{c}$, since $b_{i+1}>b_{i}$ would then be the label of a later edge. That is, if $B_{i} \mid B_{i+1}$ is non-crossing, then a descending chain has a $-b_{i}$ label. The "only if" direction is immediate, thus there is a unique permutation and set of signs for the $\pm b_{1}, \ldots, \pm b_{k-1}$ that could label a descending chain.

Proposition 3.14. Let $\mathbf{c}$ be a (non-maximal) chain with extensions in both $\Sigma_{\alpha}$ and $\Sigma_{\beta}$, $\beta<_{\text {rr-lex }} \alpha$. Then $\mathbf{c}$ has maximal extensions in $\mathcal{M}\left(\Sigma_{\alpha}\right) \backslash \mathcal{M}\left(\Delta_{\alpha}\right)$.

Proof. Let $\mathbf{c}=\left\{\hat{1}=c_{0}>c_{1} \cdots>c_{m}>c_{m+1}=\hat{0}\right\}$. The first $\beta$ with $\mathbf{c}$ in $\Sigma_{\beta}$ obeys the following two conditions:

1. For each $c_{i} \neq \hat{0}$, each block $B$ in $c_{i-1}$ splits into sub-blocks $B_{1}, \ldots, B_{k}$ in $c_{i}$, where $\max B_{1}<\cdots<\max B_{k}$. The restriction $\left.\beta\right|_{B}$ is of the form $B_{1} B_{2} \ldots B_{k}$. (By repeated application of Corollary 3.11 .)

2. For each block $B$ of $c_{m},\left.\beta\right|_{B}$ is the permutation

$$
\left\{b_{1} b_{2} \ldots b_{d+1} b_{d} \ldots b_{i d+1} b_{i d} \ldots b_{k}\right\}
$$

where $B=\left\{b_{1}, \ldots, b_{k}\right\}$ for $b_{1}<\cdots<b_{k}$. That is, $\left.\beta\right|_{B}$ is the ascending permutation of the elements of $B$, with transpositions applied at $d$-divisible positions. (The proof is by starting at the end and working to the front, greedily taking the greatest possible element for each position.)

Since $\alpha$ is not the first permutation in $A_{n}^{d}$ such that $\mathbf{c} \in \Sigma_{\alpha}, \alpha$ must violate at least one of these. If it violates (1) for some $B$, then $\left.\alpha\right|_{B}$ has the form $B_{1} \ldots B_{k}$ with $\max B_{j}>$ $\max B_{j+1}$. Merge $B_{j}$ and $B_{j+1}$ to add an edge down from $c_{i-1}$ that is in $\Sigma_{\alpha}$, otherwise extend arbitrarily in $\Sigma_{\alpha}$. By Corollary 3.11 part 1 , the resulting chain is not in $\Delta_{\alpha}$.

If $\left.\alpha\right|_{B}$ violates (2) for some $B$, then extend $\mathbf{c}$ by switch-and-splitting at every $d$ divisible position of $B$, otherwise arbitrarily in $\Sigma_{\alpha}$. At the bottom, $B$ is partitioned into some $B_{1}|\ldots| B_{k}$. Since (2) is violated, applying transpositions to $\alpha$ at $d$-divisible partitions gives a descent. But this contradicts the conclusion of Corollary 3.11 part 2, and the resulting chain is not in $\Delta_{\alpha}$.

We check the $C L$-ced properties: Wachs had already proved (CLced-polytope) as presented in Theorem 3.1, Lemma 3.10 gives us (CLced-union), Proposition 3.12 gives (CLced-desc), and Proposition 3.14 gives (CLced-bdry). We have completed the proof of Theorem 3.8. 


\section{The coset lattice}

\subsection{Group theory background}

The coset poset of $G$, denoted $\overline{\mathfrak{C}}(G)$, is the set of all right cosets of all proper subgroups of $G$, ordered under inclusion. The coset lattice of $G$, denoted $\mathfrak{C}(G)$, is $\overline{\mathfrak{C}}(G) \cup\{\emptyset, G\}$, that is, $\overline{\mathfrak{C}}(G)$ with a top $\hat{1}=G$ and bottom $\hat{0}=\emptyset$ added. With our definitions, it makes sense to look at the order complex of $\mathfrak{C}(G)$ (which is the set of all chains of $\overline{\mathfrak{C}}(G)$ ), and so we talk about the coset lattice, even though "coset poset" has a better sound to it. We notice that $\mathfrak{C}(G)$ has meet operation $H x \wedge K y=H x \cap K y$ and join $H x \vee K y=\left\langle H, K, x y^{-1}\right\rangle y$ (so it really is a lattice.) General background on the coset lattice can be found in $[22$, Chapter 8.4], and its topological combinatorics have been studied in [5, 19, 36].

The subgroup lattice of $G$, denoted $L(G)$ is the set of all subgroups of $G$, ordered by inclusion. General background can be found in [22], and its topological combinatorics have been studied extensively, for example in [24,32].

Notice that for any $x \in G$, the interval $[x, G]$ in $\mathfrak{C}(G)$ is isomorphic to $L(G)$. It is a theorem of Iwasawa [11] that $L(G)$ is graded if and only if $G$ is supersolvable, hence $\mathfrak{C}(G)$ is graded under the same conditions. As we have only defined convex ear decompositions for pure complexes, we are primarily interested in supersolvable groups in this paper.

Schweig proved the following:

Proposition 4.1. (Schweig [23]) For a supersolvable lattice L, the following are equivalent:

1. L has a convex ear decomposition.

2. L is doubly Cohen-Macaulay.

3. Every interval of $L$ is complemented.

Note 4.2. A construction very much like Schweig's convex ear decomposition was earlier used by Thévenaz in [32] on a subposet of $L(G)$ to understand the homotopy type and the conjugation action on homology of $L(G)$ for a solvable group $G$.

It is easy to check that any normal subgroup $N \triangleleft G$ is left modular in $L(G)$, so a supersolvable group has a supersolvable subgroup lattice with any chief series as its left modular chain. Let $G^{\prime}$ denote the commutator subgroup of $G$. The following collected classification of groups with every interval in their subgroup lattice complemented is presented in Schmidt's book [22, Chapter 3.3], and was worked out over several years by Zacher, Menegazzo, and Emaldi.

Proposition 4.3. The following are equivalent for a (finite) group $G$ :

1. Every interval of $L(G)$ is complemented.

2. If $H$ is any subgroup on the interval $\left[H_{0}, H_{1}\right]$, then there is a $K$ such that $H K=H_{1}$ and $H \cap K=H_{0}$. 
3. $L(G)$ is coatomic, i.e., every subgroup $H$ of $G$ is an intersection of maximal subgroups of $G$.

4. G has elementary abelian Sylow subgroups, and if $H_{1} \triangleleft H_{2} \triangleleft H_{3} \subseteq G$, then $H_{1} \triangleleft H_{3}$.

5. $G^{\prime}$ and $G / G^{\prime}$ are both elementary abelian, $G^{\prime}$ is a Hall $\pi$-subgroup of $G$, and every subgroup of $G^{\prime}$ is normal in $G$.

Note 4.4. The classification of finite simple groups is used in the proof that (3) is equivalent to the others.

We will follow Schmidt and call such a group a relatively complemented group.

We notice that relatively complemented groups are complemented, that is, satisfy the condition of Proposition 4.3 Part (2) on the interval $[1, G]$. On the other hand, $S_{3} \times \mathbb{Z}_{3}$ is an example of a complemented group which is not relatively complemented. The complemented groups are exactly the groups with (equivalently in this case) shellable, CohenMacaulay, and sequentially Cohen-Macaulay coset lattice [36]. Computation with GAP [10] shows that there are 92804 groups of order up to 511, but only 1366 complemented groups, and 1186 relatively complemented groups.

We summarize the situation for the subgroup lattice regarding convex ear decompositions:

Corollary 4.5. The following are equivalent for a group $G$ :

1. $L(G)$ has a convex ear decomposition.

2. $L(G)$ is doubly Cohen-Macaulay.

3. $G$ is a relatively complemented group.

As a consequence, we get one direction of Theorem 1.2.

Corollary 4.6. If $\mathfrak{C}(G)$ is doubly Cohen-Macaulay (hence if it has a convex ear decomposition), then $G$ is a relatively complemented group.

Proof. Every interval of a 2-Cohen-Macaulay poset is 2-Cohen-Macaulay, and the interval $[1, G]$ in $\mathfrak{C}(G)$ is isomorphic to $L(G)$.

The remainder of Section 4 will be devoted to proving the other direction.

\subsection{A dual $E L$-labeling for $\mathfrak{C}(G)$}

As with the $d$-divisible partition lattice, the first thing we need is a dual $E L$-labeling of $\mathfrak{C}(G)$. We will construct one for the more general case where $G$ is complemented. The main idea is to start with the EL-labeling of an upper interval and "twist" by adding signs, similarly to our $E L$-labeling for $\Pi_{n}^{d}$. The resulting labeling is significantly simpler than the one I described in [36]. 
Let $G$ be a complemented group, and fix a chief series $G=N_{1} \triangleright N_{2} \triangleright \cdots \triangleright N_{k+1}=1$ for $G$ throughout the remainder of Section 4. Our labeling (and later our convex ear decomposition) will depend on this choice of chief series, but the consequences for the topology and $h$-vector of $\mathfrak{C}(G)$ will obviously depend only on $G$.

For each factor $N_{i} / N_{i+1}$, choose a complement $B_{i}^{0}$, i.e., a subgroup such that $N_{i} B_{i}^{0}=G$ but $N_{i} \cap B_{i}^{0}=N_{i+1}$. (Such a $B_{i}^{0}$ exists, as every quotient group of a complemented group is itself complemented [22, Lemma 3.2.1].) From Section 2.3, the usual dual EL-labeling of the subgroup lattice of a supersolvable group is

$$
\lambda^{s s}\left(K_{0} \supset K_{1}\right)=\max \left\{i: N_{i} K_{1} \supseteq K_{0}\right\}=\min \left\{i: N_{i+1} \cap K_{0} \subseteq K_{1}\right\} .
$$

Remember that $\lambda^{s s}$ labels every chain on a given interval with the same set of labels (up to permutation).

We now define a labeling $\lambda$ of $\mathfrak{C}(G)$ as follows. For $K_{0} \supset K_{1}$ labeled by $\lambda^{s s}$ with $i$, let

$$
\begin{aligned}
\lambda\left(K_{0} x \supset K_{1} x\right) & =\left\{\begin{aligned}
-i & \text { if } K_{1} x=K_{0} x \cap B_{i}^{0}, \\
i & \text { otherwise, and }
\end{aligned}\right. \\
\lambda(x \supset \emptyset) & =0 .
\end{aligned}
$$

It is immediate from this construction that $|\lambda|_{[x, G]}=\lambda^{s s}$ (up to the "dropping $x$ " isomorphism), much like the situation discussed in Section 3.1 for the $d$-divisible partition lattice.

Lemma 4.7. Let $G$ be any supersolvable group. Then:

1. If $K B=G$ where $B \subset G$, then $K \cap B \subset K$.

2. If $\lambda^{s s}\left(K_{0} \supset K_{1}\right)=i$, then for any complement $B_{i}$ of $N_{i} / N_{i+1}$ and $K \supseteq K_{0}$ we have $K_{0} B_{i}=K B_{i}=G$.

Proof. For part 1, count: $|K \cap B|=\frac{|K||B|}{|G|}=\frac{|K|}{[G: B]}$ and by supersolvability, $[K: K \cap B]=$ $[G: B]$ is a prime.

For part 2, by the definition of the labeling, $N_{i} \cap K_{0} \nsubseteq K_{1}$ but $N_{i+1} \cap K_{0} \subseteq K_{1}$. We see that $N_{i} \cap K_{0} \nsubseteq N_{i+1}$, and since $N_{i+1} \subset N_{i}$, that $\left(N_{i} \cap K_{0}\right) N_{i+1}=N_{i}$ and so $K_{0} N_{i+1} \supseteq N_{i}$. Then $K_{0} B_{i}=K_{0} N_{i+1} B_{i} \supseteq N_{i} B_{i}=G$.

Theorem 4.8. If $G$ is a complemented group, then $\lambda$ is a dual EL-labeling of $\mathfrak{C}(G)$.

Proof. We need to show that every interval has a unique increasing maximal chain which is lexicographically first. There are two kinds of intervals we need to check:

Case 1. $\left[\emptyset, H_{0} x\right]$

As the last label of any chain on this interval is 0 , in an increasing chain the others must be negative (in increasing order). Since every chain has the same labels up to permutation, uniqueness of the increasing chain is clear from the definition of $\lambda$. Existence follows from applying Lemma 4.7 to the maximal subgroups $B_{i}^{0}$. Finally, the chain takes the edge with the least possible label down from each $H x$, so it is lexicographically first. 
Case 2. $\left[H_{n} x, H_{0} x\right]$

Let $S$ be the label set of $\lambda^{s s}$ restricted to the interval $\left[H_{n}, H_{0}\right]$. We notice that a $-i$ label is possible on $\left[H_{n} x, H_{0} x\right]$ only if $H_{n} x \subseteq B_{i}^{0}$ and $i \in S$. Thus, the lexicographically first chain is labeled by all possible negative labels (in increasing order), followed by the remaining (positive) labels, also in increasing order. Such a chain clearly exists and is increasing. The negative-labeled part is unique since a $-i$ label corresponds with intersection by $B_{i}^{0}$, while the positive-labeled part is unique since $\lambda^{s s}=|\lambda|$ is an $E L$-labeling.

It remains to check that there are no other increasing chains. We have already shown that there is only one increasing chain which has a $-i$ label for each $B_{i}^{0}$ containing $H_{n} x$, so any other increasing chain would need to have a $+i$ label for some $i \in S$ where $H_{n} x \subseteq B_{i}^{0}$. Without loss of generality, let this edge $H_{0} x \supset H_{1} x$ be directly down from $H_{0} x$. Then $i=\min S$, and since $\lambda^{s s}$ is a dual $E L$-labeling, we have that there is a unique edge down from $H_{0} x$ with label $\pm i$. But then $H_{1} x=H_{0} x \cap B_{i}^{0}$, so the edge gets a $-i$ label, giving us a contradiction and completing the proof.

Though we do not need it for our convex ear decomposition, let us briefly sketch the decreasing chains of $\lambda$. Following Thévenaz [32], a chain of complements to a chief series $G=N_{1} \supset N_{2} \supset \ldots \supset N_{k+1}=1$ is a chain of subgroups $G=H_{k+1} \supset H_{k} \supset \ldots \supset$ $H_{1}=1$ where for each $i, H_{i}$ is a complement to $N_{i}$. Thévenaz showed that the chains of complements in $G$ correspond to homotopy spheres in $|L(G)|$. The following proposition is the $E L$-shelling version of Thévenaz's result for a supersolvable group, and is a special case of [37, Proposition 4.3].

Proposition 4.9. The decreasing chains in $L(G)$ with respect to $\lambda^{\text {ss }}$ are the chains of complements to $G=N_{1} \triangleright \ldots \triangleright N_{k+1}=1$.

Proof. If $G=H_{k+1} \supset H_{k} \supset \ldots \supset H_{1}=1$ is a chain of complements, then $N_{i} H_{i}=G \supseteq$ $H_{i+1}$, while

$$
N_{i+1} H_{i} \cap H_{i+1}=\left(N_{i+1} \cap H_{i+1}\right) H_{i}=1 \cdot H_{i}=H_{i}
$$

by left modularity (the Dedekind identity). Thus $\lambda^{s s}\left(H_{i+1} \supset H_{i}\right)=i$, and the chain is descending.

Conversely, any descending chain corresponds to a sphere in $|L(G)|$, and by Thévenaz's correspondence, there can be no others.

Corollary 4.10. The decreasing chains in $\mathfrak{C}(G)$ with respect to $\lambda$ are all cosets of chains of complements $\left\{G=H_{k+1} x \supset \ldots \supset H_{1} x=x \supset \emptyset\right\}$ to the chief series $G=N_{1} \triangleright \ldots \triangleright$ $N_{k+1}=1$ such that no $H_{i} x=H_{i+1} x \cap B_{i}^{0}$.

\subsection{A convex ear decomposition for $\mathfrak{C}(G)$}

Recall that subgroups $H$ and $K$ commute if $H K=K H$ is a subgroup of $G$.

Lemma 4.11. (Warm-up Lemma) Let $G$ be a solvable group with chief series $G=N_{1} \triangleright$ $N_{2} \triangleright \cdots \triangleright N_{k+1}=1$, and $B_{i}$ and $B_{j}$ be complements of normal factors $N_{i} / N_{i+1}$ and $N_{j} / N_{j+1}$ where $i \neq j$. Then $B_{i}$ and $B_{j}$ commute. 
Proof. Suppose $j<i$. Then $N_{i+1} \subsetneq N_{i} \subseteq N_{j+1} \subsetneq N_{j}$, and $N_{j+1} \subseteq B_{j}$. Thus, $B_{j} B_{i} \supseteq$ $N_{i} B_{i}=G$.

Recalling $G=N_{1} \triangleright N_{2} \triangleright \cdots \triangleright N_{k+1}=1$ as the chief series we fixed in Section 4.2, let

$$
\mathcal{B}=\left\{B_{i}: B_{i} \text { is a complement to } N_{i} / N_{i+1}, 1 \leq i \leq k\right\}
$$

be a set of complements to $N_{i}$, one complement for each chief factor (so that $|\mathcal{B}|=k$ ). For any $x \in G$, let $\mathcal{B} x=\left\{B_{i} x: B_{i} \in \mathcal{B}\right\}$. We will call $\mathcal{B}$ a base-set for $\mathfrak{C}(G)$.

The first step is to show that intersections of certain cosets of $\mathcal{B}$ give us a cube, using a stronger version of Lemma 4.11 .

Lemma 4.12. If $\mathcal{B}$ is a base-set, then $\left(B_{i_{1}} \cap \cdots \cap B_{i_{l}}\right) B_{i_{\ell+1}}=G$.

Proof. We count

$$
\begin{aligned}
\left|\left(B_{i_{1}} \cap \cdots \cap B_{i_{\ell}}\right) B_{i_{\ell+1}}\right| & =\frac{\left|\left(B_{i_{1}} \cap \cdots \cap B_{i_{\ell}}\right)\right|\left|B_{i_{\ell+1}}\right|}{\left|B_{i_{1}} \cap \cdots \cap B_{i_{\ell}} \cap B_{i_{\ell+1}}\right|} \\
& =\frac{\left|B_{i_{1}} \cap \cdots \cap B_{i_{\ell-1}}\right|\left|B_{i_{\ell}}\right|\left|B_{i_{\ell+1}}\right|}{\left|\left(B_{i_{1}} \cap \cdots \cap B_{i_{\ell-1}}\right) B_{i_{\ell}}\right|\left|B_{i_{1}} \cap \cdots \cap B_{i_{\ell}} \cap B_{i_{\ell+1}}\right|}
\end{aligned}
$$

By induction on $\ell$, this is

$$
=\frac{\left|B_{i_{1}} \cap \cdots \cap B_{i_{\ell-1}}\right|\left|B_{i_{\ell}}\right|\left|B_{i_{\ell+1}}\right|}{|G|\left|B_{i_{1}} \cap \cdots \cap B_{i_{\ell}} \cap B_{i_{\ell+1}}\right|}
$$

and by symmetry,

$$
\left|\left(B_{i_{1}} \cap \cdots \cap B_{i_{\ell}}\right) B_{i_{\ell+1}}\right|=\left|\left(B_{i_{1}} \cap \cdots \cap B_{i_{\ell-1}} \cap B_{i_{\ell+1}}\right) B_{i_{\ell}}\right| .
$$

Repeating this argument shows that $\left|\left(B_{i_{1}} \cap \cdots \cap B_{i_{\ell}}\right) B_{i_{\ell+1}}\right|$ is independent of the ordering of the $B_{i_{j}}$ 's, or of the choice of $i_{\ell+1}$.

Then take $i_{\ell+1}$ to be the largest index of any such $B_{i_{j}}$, so that $N_{i_{\ell+1}} \subseteq B_{i_{1}} \cap \cdots \cap B_{i_{\ell}}$. In particular, $\left(B_{i_{1}} \cap \cdots \cap B_{i_{\ell}}\right) B_{i_{\ell+1}} \supseteq N_{i_{\ell+1}} B_{i_{\ell+1}}=G$. Since the ordering of the $i_{j}$ 's doesn't affect the cardinality, $\left|\left(B_{i_{1}} \cap \cdots \cap B_{i_{\ell}}\right) B_{i_{\ell+1}}\right|=|G|$ for any choice of $i_{\ell+1}$, proving the lemma.

Corollary 4.13. If $\mathcal{B}$ is a base-set and $x$ is such that the elements of $\mathcal{B}$ and $\mathcal{B} x$ are distinct from one another (i.e., $B_{i} \neq B_{i} x$ for all $i$ ), then the meet sublattice generated by $\mathcal{B} \cup \mathcal{B} x$ is isomorphic to the face lattice of the boundary of a $k$-cube.

Proof. Any $B_{j}$ commutes with any intersection of $B_{i}$ 's, $j \neq i$, and the result follows from Lemma 4.7 and since $B_{i} \cap B_{i} x=\emptyset$ for all $i$. 
We henceforth assume that $G$ is relatively complemented.

Let $\mathcal{B}$ be a base-set for $\mathfrak{C}(G)$ as above, and $x \in G$ be such that $B_{i} x \neq B_{i}^{0}$ (for each $i$ ). Then we define $\Sigma_{\mathcal{B} x}$ to be the meet sublattice of $\mathfrak{C}(G)$ generated by $\mathcal{B} x \cup\left\{B_{i}^{0}: B_{i} x=\right.$ $\left.B_{i}^{0} x\right\}$, and the larger meet sublattice $\Sigma_{\mathcal{B} x}^{+}$to be generated by

$$
\mathcal{B} x \cup\left\{B_{i}^{0}: B_{i} x=B_{i}^{0} x\right\} \cup\left\{B_{i} y_{i}: B_{i} \neq B_{i}^{0}\right\},
$$

where the $y_{i}$ 's are some elements such that $B_{i} y_{i} \neq B_{i} x$. By Lemma 4.7 and the proof of Corollary 4.13,

$$
\bigcap_{\left\{i: B_{i} x=B_{i}^{0} x\right\}} B_{i}^{0} \cap \bigcap_{\left\{i: B_{i} \neq B_{i}^{0}\right\}} B_{i} y_{i}=y \text { (for some } y \text { ), }
$$

so $\Sigma_{\mathcal{B} x}^{+}$is given by all intersections of $\mathcal{B} x \cup \mathcal{B} y$. Thus (also by Corollary 4.13) $\left|\Sigma_{\mathcal{B} x}^{+}\right|$is a convex polytope with subcomplex $\left|\Sigma_{\mathcal{B} x}\right|$.

Lemma 4.14. Let $H_{0} x \supset H_{1} x$ be an edge in $\mathfrak{C}(G)$ with $\lambda\left(H_{0} x \supset H_{1} x\right)=i$. Then $H_{1} x=H_{0} x \cap B_{i} x$ for some complement $B_{i}$ to $N_{i} / N_{i+1}$.

Proof. Since every maximal chain in $\mathfrak{C}(G)$ has exactly one edge with $\lambda\left(H_{0} x \supset H_{1} x\right)= \pm i$ for each $i \in[k]$, it suffices to show that $H_{1}$ is contained in some complement $B_{i}$ to $N_{i} / N_{i+1}$. Then $H_{0}$ cannot be contained in $B_{i}$, as that would give two $\pm i$ edges, and so $H_{1}=H_{0} \cap B_{i}$.

Since $G$ is relatively complemented, every interval in $L(G)$ is complemented. In particular, any interval of height 2 has both increasing and decreasing chains, so for any $H_{-1} \supset H_{0}$ there is an $H_{1}^{+} \subset H_{-1}$ with $\lambda^{s s}\left(H_{-1} \supset H_{1}^{+}\right)=i$.

Repeat this argument inductively on $H_{-1} \supset H_{1}^{+}$until $H_{-1}=G$. The final $H_{1}^{+}$is the desired $B_{i}$, and the definition of $\lambda^{s s}$ shows that $B_{i}$ is a complement to $N_{i} / N_{i+1}$.

Corollary 4.15. Every maximal chain in $\mathfrak{C}(G)$ is in some $\Sigma_{\mathcal{B} x}$.

Corollary 4.15 would not hold if we replaced 'relatively complemented' with any weaker condition, since the result implies coatomicity, and Proposition 4.3 tells us that relatively complemented groups are exactly those with coatomic subgroup lattice.

Now that we have a set of cubes that cover $\mathfrak{C}(G)$, the next step is to assign an order to them. For any base-set $\mathcal{B}$, let $\rho_{i}(\mathcal{B})$ be 0 if $B_{i}=B_{i}^{0}$, and 1 otherwise. We put the $\rho_{i}$ 's together in a binary vector $\rho(\mathcal{B})$, which we will call the pattern of $\mathcal{B}$. Order the $\mathcal{B} x$ 's (and hence the $\Sigma_{\mathcal{B} x}$ 's) in any linear extension of the lexicographic order on $\rho(\mathcal{B})$. Let $\Delta_{\mathcal{B} x}$ be the simplicial complex with facets the maximal chains that are in $\Sigma_{\mathcal{B} x}$, but not in any preceding $\Sigma_{\mathcal{B}^{\prime} x^{\prime}}$.

The $\Sigma_{\mathcal{B} x}$ 's are generally proper subsets of face lattices of convex polytopes, so (CLcedpolytope) does not hold and we do not have an $E L$-ced. We can use the same sort of argument, however, to prove the following refinement of Theorem 1.2:

Theorem 4.16. $\left\{\Delta_{\mathcal{B} x}\right\}$ is a convex ear decomposition for $\mathfrak{C}(G)$ under the pattern ordering.

Corollary 4.15 shows that the ears cover $\mathfrak{C}(G)$, that is, that (CLced-union) holds. Our next step is to show that an analogue of (CLced-desc) holds.

It will be convenient to let $S([a, b])$ be the label set of $|\lambda|$ on the interval $[a, b]$, that is, the set of nonnegative $i$ 's such that $\lambda$ gives $\pm i$ labels on cover relations in $[a, b]$. 
Lemma 4.17. For any interval $[a, b]$ in $\mathfrak{C}(G)$, there is at most one (dual) descending maximal chain $\mathbf{c}$ on $[a, b]$ which is in $\Delta_{\mathcal{B} x}$.

Proof. If $a=\emptyset$, then the unique descending chain on $[a, b]$ in $\Sigma_{\mathcal{B} x}$ is given by intersecting with each $B_{i} x(i \in S([\emptyset, b]) \backslash\{0\})$ in order.

If $a \neq \emptyset$, then the interval $[a, b]$ in $\Sigma_{\mathcal{B} x}$ is Boolean, with a maximal chain for each permutation of $S([a, b])$. If there is a $-i$ label on a chain in $\Delta_{\mathcal{B} x}$, then the edge can be obtained by intersecting with $B_{i}^{0}$. But since $\Sigma_{\mathcal{B} x}$ is the first such complex containing the chain, we must have $\rho_{i}(\mathcal{B})=0$ (otherwise, replace $B_{i} x$ with $B_{i}^{0}$ ). But this tells us that every label with absolute value $i$ on $[a, b]$ in $\Delta_{\mathcal{B} x}$ is negative. Every such chain thus has the same set of labels, and at most one permutation of these labels is descending.

Corollary 4.18. $\Delta_{\mathcal{B} x}$ is shellable.

Proof. Suppose a maximal chain $\mathbf{c}=\left\{G=c_{1} \supset \ldots \supset c_{k+1} \supset c_{k+2}=\emptyset\right\}$ in $\Sigma_{\mathcal{B} x}$ has an ascent at $j$. If $j \neq k+1$, then it is immediate that $\mathbf{c} \backslash\left\{c_{j}\right\}$ has two extensions in $\Sigma_{\mathcal{B} x}$, and we argue exactly as in Lemma 2.7 and Theorem 2.9.

If $j=k+1$, then the ascent at $j$ has labels $-i, 0$, and hence $\rho_{i}(\mathcal{B})=0$ and $\Sigma_{\mathcal{B} x}$ is the first cube containing $\mathbf{c} \backslash\left\{c_{k+1}\right\}$. Intersecting with $B_{i}^{0} x$ instead of $B_{i}^{0}$ at $c_{k}$ gives another chain $\mathbf{c}^{\prime}$ in $\Delta_{\mathcal{B} x}$ with a descent at $k+1$, and we again argue as in Theorem 2.9.

Finally, we show directly that (ced-bdry) holds. We start with a lemma.

Lemma 4.19. Given any chain $\mathbf{c}=\left\{G=c_{1} \supset \cdots \supset c_{m} \supset c_{m+1}=\emptyset\right\}$, there is an extension to a maximal chain $\mathbf{c}^{++}$such that if $\mathbf{c}$ is in $\Sigma_{\mathcal{B} x}$, then $\mathbf{c}^{++}$is in some $\Sigma_{\mathcal{B} x}^{+}$. If $\mathcal{B} x$ is the first such with $\mathbf{c}$ in $\Sigma_{\mathcal{B} x}$, then $\mathbf{c}^{++}$is in $\Sigma_{\mathcal{B} x}$.

Proof. We make the extension in two steps. First, let $\mathbf{c}^{+}$be the extension of $\mathbf{c}$ by augmenting each $c_{j} \supset c_{j+1}$ for $j \neq m$ with the chain on $\left[c_{j+1}, c_{j}\right]$ that is increasing according to $|\lambda|$. Intersecting $c_{j}$ iteratively with $B_{i} x$ or $B_{i}^{0}$ (as appropriate, for each $i$ in $S\left(\left[c_{j+1}, c_{j}\right]\right)$ ) in increasing order gives this chain, thus, $\mathbf{c}^{+}$is also in $\Sigma_{\mathcal{B} x}$.

In a similar manner, let $\mathbf{c}^{++}$be the extension of $\mathbf{c}^{+}$at $c_{m} \supset \emptyset$ by intersecting with each $B_{i}^{0}$ for $i \in S(m)$ in increasing order. Suppose $c_{m}=H x$. Then uniqueness of the lexicographically first chain in $[1, H]$ gives that $H \cap B_{i}^{0}=H \cap B_{i}$, so there is some $B_{i} y_{i}$ with $H x \cap B_{i}^{0}=H x \cap B_{i} y_{i}$. Repeated use of this gives us a $\Sigma_{\mathcal{B} x}^{+}$containing $\mathbf{c}^{++}$: the generating elements for this cube include those for $\Sigma_{\mathcal{B} x}$ and the $B_{i} y_{i}$ 's found here. Notice that if $\rho(i)=0$ for each $i \in S\left(\left[\emptyset, c_{m}\right]\right)$, then $B_{i}^{0}$ is already in the generating set for $\Sigma_{\mathcal{B} x}$, thus $\mathbf{c}^{++}$is also in $\Sigma_{\mathcal{B} x}$.

Proposition 4.20. $\Delta_{\mathcal{B} x} \cap\left(\bigcup_{\mathcal{B}^{\prime} x^{\prime} \prec \mathcal{B} x} \Delta_{\mathcal{B}^{\prime} x^{\prime}}\right)=\partial \Delta_{\mathcal{B} x}$.

Proof. Suppose that $\mathbf{c}$ is in $\Delta_{\mathcal{B} x} \cap\left(\bigcup_{\mathcal{B}^{\prime} x^{\prime} \prec \mathcal{B} x} \Delta_{\mathcal{B}^{\prime} x^{\prime}}\right)$, and let $\mathbf{c}^{++}$be as in Lemma 4.19. Then $\mathbf{c}^{++}$is an extension in $\Sigma_{\mathcal{B} x}^{+}$, but since $\mathbf{c}^{++}$is contained in $\Sigma_{\mathcal{B}^{\prime} x}$ for the first such complex containing $\mathbf{c}$, we get that $\mathbf{c}^{++}$is in $\mathcal{M}\left(\Sigma_{\mathcal{B} x}^{+}\right) \backslash \mathcal{M}\left(\Delta_{\mathcal{B} x}\right)$. Lemma 2.8 then gives that $\mathbf{c}$ is in $\partial \Delta_{\mathcal{B} x}$.

Conversely, let $\mathbf{c}$ be in $\Delta_{\mathcal{B} x}$, but not in a previous $\Sigma_{\mathcal{B}^{\prime} x^{\prime}}$. Since $\mathbf{c}$ is not in any previous $\Sigma_{\mathcal{B}^{\prime} x^{\prime}}$, no extensions of it are either, so any extension of $\mathbf{c}$ that is in $\Sigma_{\mathcal{B} x}$ is in $\Delta_{\mathcal{B} x}$. As 
we have ordered the base-sets by pattern, we get that $\rho_{i}(\mathcal{B})=0$ for $i \in S\left(\left[\emptyset, c_{m}\right]\right)$, thus, by the special treatment of $B_{i}^{0}$ in the definition of $\Sigma_{\mathcal{B} x}$, every extension of $\mathbf{c}$ in any $\Sigma_{\mathcal{B} x}^{+}$ is in $\Sigma_{\mathcal{B} x}$. Combining these two statements, we see that there is no extension of $\mathbf{c}$ in $\mathcal{M}\left(\Sigma_{\mathcal{B} x}^{+}\right) \backslash \mathcal{M}\left(\Delta_{\mathcal{B} x}\right)$, and so by Lemma 2.8 that $\mathbf{c}$ is not in $\partial \Delta_{\mathcal{B} x}$.

We have now finished the proof of Theorem 4.16. Let us review: Corollary 4.13 gave us (ced-polytope), Proposition 4.20 was (ced-bdry), and Corollary 4.15 gave us (ced-union). We notice that the base-set with the earliest pattern is $\mathcal{B}_{0}=\left\{B_{i}^{0}\right\}$, and that each $\Sigma_{\mathcal{B}_{0} x}$ is the face lattice of a cube. Thus the first $\Delta_{\mathcal{B} x}$ is a polytope, while all subsequent ones are proper subcomplexes of polytopes. Since we proved in Corollary 4.18 that each $\Delta_{\mathcal{B} x}$ is shellable, we have (ced-topology).

Note 4.21. As previously mentioned, the convex ear decomposition we have constructed is not a (dual) $E L$-ced. Although we would rather find an $E L$-ced than a general convex ear decomposition, this is not in general possible with the cubes we are looking at here. For example $\mathfrak{C}\left(\mathbb{Z}_{2}^{2}\right)$ has exactly three possible $\Sigma_{\mathcal{B} x}^{+}$'s, but the homotopy type of the wedge of 61 -spheres, so some $\left|\Sigma_{\mathcal{B} x}^{+}\right| \backslash\left|\Sigma_{\mathcal{B}^{\prime} x^{\prime}}^{+}\right|$must be disconnected. The example of $\mathfrak{C}\left(\mathbb{Z}_{2}^{2}\right)$ is a geometric lattice, so does have an $E L$-ced (for a different $E L$-labeling), but I have not been able to extend this to an $E L$-ced for other relatively complemented groups.

The reader may have noticed that the constructed convex ear decomposition is not far from being an $E L$-ced - the difference is that each $\Sigma_{\mathfrak{B} x}^{+}$gives several "new" ears - and that another possibility would be to extend the definition of $E L$-ced to cover this case. However, as this would make the definition more complicated, and as the gain seems relatively small, I have chosen to leave the definition as presented.

\section{$5 \quad$ Poset products}

Throughout this section, let $P_{1}$ and $P_{2}$ be bounded posets.

In Section 3.1, we defined the product $P_{1} \times P_{2}$ and lower reduced product $P_{1} \check{\times} P_{2}$ of $P_{1}$ and $P_{2}$. It should come as no surprise that the upper reduced product $P_{1} \hat{\times} P_{2}$ of $P_{1}$ and $P_{2}$ is defined as $\left(\left(P_{1} \backslash\{\hat{1}\}\right) \times\left(P_{2} \backslash\{\hat{1}\}\right)\right) \cup\{\hat{1}\}$. There is a natural inclusion of $P_{1} \check{\times} P_{2}$ (and of $\left.P_{1} \hat{\times} P_{2}\right)$ into $P_{1} \times P_{2}$.

Our goal in Section 5 is to explain the background and give proofs for Theorems 1.4 and 1.6. The flavor and techniques of this section are different from the previous two, so we pause to justify its connection with "Cubical Convex Ear Decompositions". Lower reduced products come up fundamentally both in the $d$-divisible partition lattice, as we discussed in Section 3.1, as well as in the coset lattice, where $\mathfrak{C}\left(G_{1} \times G_{2}\right) \cong \mathfrak{C}\left(G_{1}\right) \check{\times} \mathfrak{C}\left(G_{2}\right)$ for groups $G_{1}$ and $G_{2}$ of co-prime orders. And some of the decompositions in product posets are cubical after all: a cube is the direct product of intervals, so if $C_{d}$ is the boundary of the $d$-cube, with face lattice $L\left(C_{d}\right)$, then $L\left(C_{d}\right)=\check{\prod}_{1}^{d} L\left(C_{1}\right)$.

\subsection{Poset products and polytopes}

I am told that the following proposition is folklore. It is also discussed briefly in [12]. 
Proposition 5.1. If $\Sigma_{1}$ and $\Sigma_{2}$ are the face lattices of convex polytopes $X_{1}$ and $X_{2}$, then

1. $\Sigma_{1} \times \Sigma_{2}$ is the face lattice of the "free join" $X_{1} \circledast X_{2}$, a convex polytope.

2. $\Sigma_{1} \check{\times} \Sigma_{2}$ is the face lattice of the Cartesian product $X_{1} \times X_{2}$, a convex polytope.

3. $\Sigma_{1} \hat{\times} \Sigma_{2}$ is the face lattice of the "free sum" of $X_{1}$ and $X_{2}$, a convex polytope.

Proposition 5.1 guides us to a proof of Lemma 1.5. Our main tool will be stellar subdivision.

If $\Delta$ is a convex polytope with a proper face $\sigma$, then a stellar subdivision of $\Delta$ at $\sigma$, denoted $\operatorname{stellar}_{\sigma} \Delta$, is conv $\left(\Delta \cup\left\{v_{\sigma}\right\}\right)$, where $v_{\sigma}=w_{\sigma}-\varepsilon\left(w_{\Delta}-w_{\sigma}\right)$ for some point $w_{\sigma}$ in the relative interior of $\sigma$, some point $w_{\Delta}$ in the interior of $\Delta$, and a small number $\varepsilon$. In plain language, we "cone off" a new vertex lying just over $\sigma$. Note that the relative interior of a vertex is the vertex itself. Stellar subdivisions are discussed in depth in $[9$, III.2] and [8].

The main fact [9, III.2.1, III.2.2] that we will need is that the faces of the boundary complex of stellar $\sigma$ are

$$
\{\tau: \sigma \not \tau\} \cup\left\{v_{\sigma} * \tau: \tau \in \Delta \text { with } \tau, \sigma \subseteq \tau^{\prime} \text { for some } \tau^{\prime} \in \Delta \text {, but } \sigma \not \subset\right\} \text {. }
$$

Thus the stellar subdivision replaces the faces containing $\sigma$ with finer subdivisions.

Example 5.2. [8, Section 2] The barycentric subdivision of a polytopal $d$-complex $\Delta$ is the repeated stellar subdivision of $\Delta$ along a reverse linear extension of its face lattice $L(\Delta)$. That is, subdivide each $d$-dimensional face, then each $(d-1)$-dimensional face, and so forth.

If $X$ is the boundary complex of a polytope, then let $\bar{X}$ denote conv $X$, that is, the polytope of which $X$ is the boundary complex.

Lemma 5.3. Suppose $P_{1}$ and $P_{2}$ are bounded posets and that $\left|P_{1}\right|$ and $\left|P_{2}\right|$ are the boundary complexes of polytopes. Then $\left|P_{1} \check{\times} P_{2}\right|$ can be obtained from the boundary complex of $\overline{\left|P_{1}\right|} \times \overline{\left|P_{2}\right|}$ by a sequence of stellar subdivisions.

Proof. Let $\Delta_{0}$ be the boundary complex of $\overline{\left|P_{1}\right|} \times \overline{\left|P_{2}\right|}$. The faces of $\Delta_{0}$ are exactly the products $F^{(1)} \times F^{(2)}$, where each $F^{(i)}$ is a non-empty face in $P_{i}$, and at least one is proper. In particular the vertices are products of vertices $v^{(1)} \times v^{(2)}$, where $v^{(i)}$ is in $P_{i} \backslash\{\hat{0}, \hat{1}\}$. We write this product of vertices as $\left(v^{(1)}, v^{(2)}\right)$, and think of it as sitting in $\left|P_{1} \check{\times} P_{2}\right|$.

We start by ordering the elements $\left\{v^{(2)}\right\}$ of $P_{2}$ by a reverse linear extension, and stellarly subdividing at each $\sigma=\overline{\left|P_{1}\right|} \times v^{(2)}$ in this order. Inductively assume that the faces containing $\sigma$ are those of the form $\left(\overline{\left|P_{1}\right|} \times F^{(2)}\right) * C$, where $F^{(2)}$ is a face of $\left|P_{2}\right|$ with top-ranked vertex $v^{(2)}$, and $C$ is a simplex corresponding to (the simplicial join of) a chain of elements of the form $\left(\hat{1}, w^{(2)}\right)$ (with each $w^{(2)}>v^{(2)}$ ). Subdivision replaces these faces with those of the form $\left(\overline{\left|P_{1}\right|} \times F_{0}^{(2)}\right) * C *\left\{v_{\sigma}\right\}$, where $F_{0}^{(2)}$ is a face having top-ranked vertex $<v^{(2)}$. We abuse notation to call the newly introduced vertex $v_{\sigma}$ as $\left(\hat{1}, v^{(2)}\right)$, which puts us in the situation required to continue our induction. 
We next do the same procedure for the faces $v^{(1)} \times \overline{\left|P_{2}\right|}$. That is, we order $\left\{v^{(1)}\right\}$ by a reverse linear extension of $P_{1}$, and repeatedly perform stellar subdivision at each such face according to this order. Since a face cannot contain both $\overline{\left|P_{1}\right|}$ and $\overline{\left|P_{2}\right|}$, these stellar subdivisions are independent of the ones at $\overline{\left|P_{1}\right|} \times v^{(2)}$.

After subdividing at all $\overline{\left|P_{1}\right|} \times v^{(2)}$ and $v^{(1)} \times \overline{\left|P_{2}\right|}$, we obtain a complex $\Delta_{1}$. The vertex set of $\Delta_{1}$ is exactly $P_{1} \check{\times} P_{2} \backslash\{\hat{0}, \hat{1}\}$. The faces of $\Delta_{1}$ are $\left\{\left(F^{(1)} \times F^{(2)}\right) * C\right\}$, where $F^{(i)}$ is a face of $\left|P_{i}\right|$, and $C$ is a simplex corresponding to either a chain of elements $\left(\hat{1}, w^{(2)}\right)$ or a chain of elements $\left(w^{(1)}, \hat{1}\right)$.

Finally, we perform stellar subdivision at the vertices $v=\left(v^{(1)}, v^{(2)}\right)$, where $v^{(i)} \in P_{i} \backslash$ $\{\hat{0}, \hat{1}\}$, in the order of a reverse linear extension of $P_{1} \check{\times} P_{2}$. We make an induction argument parallel to the one above: at the step associated with vertex $v$, the faces containing $v$ are $\left\{\left(F^{(1)} \times F^{(2)}\right) * C\right\}$. As before, $F^{(i)}$ is a face of $\left|P_{i}\right|$ with top-ranked vertex $v^{(i)}$, and $C$ corresponds to (the simplicial join of) elements in a chain greater than $v$ in $P_{1} \check{\times} P_{2}$. Stellar subdivision at $v$ replaces these faces with $\left\{\left(F_{0}^{(1)} \times F_{0}^{(2)}\right) * C *\{v\}\right\}$, where $F_{0}^{(i)}$ has greatest vertex $<v^{(i)}$, and we continue the induction.

When we have subdivided at every vertex, we obtain a complex $\Delta_{2}$. The faces of $\Delta_{2}$ are simply $\{C\}$, where $C$ is the simplicial join of vertices in a chain of $P_{1} \check{\times} P_{2}$, which is the definition of the order complex $\left|P_{1} \check{\times} P_{2}\right|$.

Corollary 5.4. If If $P_{1}$ and $P_{2}$ are bounded posets such that $\left|P_{1}\right|$ and $\left|P_{2}\right|$ are the boundary complexes of polytopes, then $\left|P_{1} \check{\times} P_{2}\right|$ and (by duality) $\left|P_{1} \hat{\times} P_{2}\right|$ are also boundary complexes of polytopes.

For $P_{1} \times P_{2}$, a similar result holds. Recall that the free join $\Delta_{1} \circledast \Delta_{2}$ of two polytopes $\Delta_{1}$ and $\Delta_{2}$ is obtained by taking the convex hull of embeddings of $\Delta_{1}$ and $\Delta_{2}$ into skew affine subspaces of Euclidean space (of high enough dimension). The faces of $\Delta_{1} \circledast \Delta_{2}$, as hinted in Proposition 5.1, are $F^{(1)} \circledast F^{(2)}$, and $\operatorname{dim} F^{(1)} \circledast F^{(2)}=\operatorname{dim} F^{(1)}+\operatorname{dim} F^{(2)}+1$.

Lemma 5.5. Suppose $P_{1}$ and $P_{2}$ are bounded posets and that $\left|P_{1}\right|$ and $\left|P_{2}\right|$ are the boundary complexes of polytopes. Then $\left|P_{1} \times P_{2}\right|$ can be obtained from the boundary complex of $\overline{\left|P_{1}\right|} \circledast \overline{\left|P_{2}\right|}$ by a sequence of stellar subdivisions.

Proof. Since the details of the proof are very similar to the preceding Lemma 5.3, we provide a sketch only. Let $\Delta_{0}=\overline{\left|P_{1}\right|} \circledast \overline{\left|P_{2}\right|}$. Notice that the vertices of $\Delta_{0}$ are $\left\{\emptyset \circledast v^{(2)}\right\} \cup$ $\left\{v^{(1)} \circledast \emptyset\right\}$, while the edges are $\left\{v^{(1)} \circledast v^{(2)}\right\}$.

As in Lemma 5.3, we begin by ordering the facets $\overline{\left|P_{1}\right|} \circledast v^{(2)}$ and $v^{(1)} \circledast \overline{\left|P_{2}\right|}$ according to reverse linear extensions of $P_{2}$ and $P_{1}$, and inductively performing stellar subdivision. Each such subdivision creates a vertex, which we name $\left(\hat{1}, v^{(2)}\right)$ or $\left(v^{(1)}, \hat{1}\right)$. We obtain a complex $\Delta_{1}$ with faces $\left\{\left(F^{(1)} \circledast F^{(2)}\right) * C\right\}$ where $F^{(i)}$ is a proper face of $P_{i}$ (possibly empty), and $C$ corresponds to a chain in the elements $\left\{\left(\hat{1}, v^{(2)}\right)\right\}$ or $\left\{\left(v^{(1)}, \hat{1}\right)\right\}$.

We then order the edges $v^{(1)} \circledast v^{(2)}$ by a linear extension of $P_{1} \times P_{2}$, and inductively perform stellar subdivision to create vertices $\left(v^{(1)}, v^{(2)}\right)$. The resulting complex is isomorphic to $\left|P_{1} \times P_{2}\right|$.

Corollary 5.6. If $P_{1}$ and $P_{2}$ are bounded posets such that $\left|P_{1}\right|$ and $\left|P_{2}\right|$ are the boundary complexes of polytopes, then $\left|P_{1} \times P_{2}\right|$ is also the boundary complex of a polytope. 
This completes the proof of Lemma 1.5.

\subsection{Convex ear decompositions of product posets}

Let $P_{1}$ and $P_{2}$ be bounded posets with respective convex ear decompositions $\left\{\Delta_{s}^{(1)}\right\}$ and $\left\{\Delta_{t}^{(2)}\right\}$. Let $P$ be either $P_{1} \times P_{2}, P_{1} \check{x} P_{2}$, or $P_{1} \hat{\times} P_{2}$; with coordinate projection maps $p_{1}$ and $p_{2}$. Take $d=\operatorname{dim}|P|, d_{1}=\operatorname{dim}\left|P_{1}\right|$, and $d_{2}=\operatorname{dim}\left|P_{2}\right|$.

We define $\Delta_{s, t}$ to be the simplicial complex generated by the maximal chains of $P$ that project to $\Delta_{s}^{(1)}$ in the first coordinate, and $\Delta_{t}^{(2)}$ in the second. Order these complexes lexicographically by $(s, t)$.

Theorem 5.7. $\left\{\Delta_{s, t}\right\}$ is a convex ear decomposition for $|P|$.

Proof. Lemma 1.5 gives that $\Delta_{s, t}$ is a subcomplex of the boundary complex of a polytope, so (ced-polytope) is satisfied.

The topology of various poset products is nicely discussed in Sundaram's [30, Section 2]. There are homeomorphisms

$$
\left|P_{1} \check{\times} P_{2}\right| \approx\left|P_{1} \hat{\times} P_{2}\right| \approx\left|P_{1}\right| *\left|P_{2}\right|,
$$

where $*$ is the join of topological spaces. This result goes back to Quillen [18, Proposition 1.9], although his notation was much different - Sundaram makes the connection in [30, proof of Proposition 2.5]. Walker [34, Theorem 5.1 (d)] extends this to show that

$$
\left|P_{1} \times P_{2}\right| \approx \operatorname{susp}\left(\left|P_{1}\right| *\left|P_{2}\right|\right),
$$

where susp denotes the topological suspension. Identical proofs to Quillen's and Walker's show that $\Delta_{s, t} \approx \Delta_{s} * \Delta_{t}$ in the upper/lower reduced case, and that $\Delta_{s, t} \approx \operatorname{susp}\left(\Delta_{s} * \Delta_{t}\right)$ in the direct product case. In particular, $\Delta_{s, t}$ is a $d$-ball for $(s, t)>(1,1)$ and a $d$-sphere for $(s, t)=(1,1)$ by results in PL-topology [20, Proposition 2.23]. We have shown that (ced-topology) is satisfied.

It is clear that (ced-union) holds. It remains to check (ced-bdry).

Claim 5.8. $\partial \Delta_{s, t}$ is exactly the set of all faces in $\Delta_{s, t}$ that project to either $\partial \Delta_{s}^{(1)}$ or $\partial \Delta_{t}^{(2)}$ (or both).

Proof. The boundary of a simplicial $d$-ball $\Delta$ is generated by the $d-1$ faces that are contained in only a single facet of $\Delta$. If $\mathbf{c}$ is a $d-1$ face of $\Delta_{s, t}$ (i.e., a chain of length $d-1)$, then at least one of $p_{1}(\mathbf{c})$ and $p_{2}(\mathbf{c})$ also has codimension 1 .

Since $\Delta_{s, t}$ is defined to be the chains which project to $\Delta_{s}^{(1)}$ and $\Delta_{t}^{(2)}$, we see that if $p_{1}(\mathbf{c})$ is $d_{1}-1$ dimensional and $\mathbf{c}$ is $d-1$ dimensional, then $p_{1}(\mathbf{c})$ has exactly one extension in $\Delta_{s}^{(1)}$ if and only if $\mathbf{c}$ has exactly one extension in $\Delta_{s, t}$. The argument if $p_{2}(\mathbf{c})$ has codimension 1 is entirely similar. 
We now show both inclusions for (ced-bdry). If $\mathbf{d}$ is any chain in $\partial \Delta_{s, t}$ with $p_{1}(\mathbf{d})$ in $\partial \Delta_{s}^{(1)}$, then $p_{1}(\mathbf{d})$ is in $\Delta_{u}^{(1)}$ for some $u<s$ by (ced-bdry), so $\mathbf{d}$ is in $\Delta_{u, t}$; similarly if $p_{1}(\mathbf{d})$ is maximal and $p_{2}(\mathbf{d})$ is in $\partial \Delta_{t}^{(2)}$. Thus $\partial \Delta_{s, t} \subseteq \Delta_{s, t} \cap\left(\bigcup_{(u, v)<(s, t)} \Delta_{u, v}\right)$.

In the other direction: if $\mathbf{c}$ is in $\Delta_{s, t}$ and $\Delta_{u, v}$ (for $(u, v)<(s, t)$ ), then $p_{1}(\mathbf{c})$ is in both $\Delta_{s}^{(1)}$ and $\Delta_{u}^{(1)}$. If $s \neq u$, then $p_{1}(\mathbf{c})$ is in $\partial \Delta_{s}^{(1)}$, so $\mathbf{c}$ is in $\partial \Delta_{s, t}$. A similar argument

applies for $p_{2}$ when $s=u$. Thus, $\partial \Delta_{s, t} \supseteq \Delta_{s, t} \cap\left(\bigcup_{(u, v)<(s, t)} \Delta_{u, v}\right)$, and we have shown (ced-bdry), completing the proof.

\subsection{Product $C L$-labelings}

In this subsection, we explicitly recall the product $C L$-labelings introduced by Björner and Wachs in [4, Section 10], and hinted at in Section 3.1. Since there is no particular reason to work with dual labelings in Section 5, I've chosen to work with standard (not dual) $C L$-labelings, so that everything is "upside down" relative to Sections 3 and 4 . Since the root of an edge of the form $\hat{0} \lessdot x$ is always $\emptyset$, we suppress the root from our notation in this case.

Let $P$ be a bounded poset with a $C L$-labeling $\lambda$ that has label set $S_{\lambda}$. A label $s \in S_{\lambda}$ is atomic if it is used to label a cover relation $\hat{0} \lessdot x$ (for any atom $x$ ), and non-atomic if it is used to label any other rooted cover relation. (In an arbitrary $C L$-labeling, a label can be both atomic and non-atomic.) A $C L$-labeling is orderly if $S_{\lambda}$ is totally ordered and partitions into $S_{\lambda}^{-}<S_{\lambda}^{A}<S_{\lambda}^{+}$, where every atomic label is in $S_{\lambda}^{A}$, and every non-atomic label is either in $S_{\lambda}^{-}$or $S_{\lambda}^{+}$. There are similar definitions of co-atomic, non-co-atomic, and co-orderly, and of course we can generalize to talk of orderly and co-orderly chain edge labelings, even if the $C L$-property is not met.

Lemma 5.9. (Björner and Wachs [4, Lemma 10.18]) Let $P$ be a bounded poset with a $C L$-labeling $\lambda$. Then $P$ has an orderly $C L$-labeling $\lambda^{\prime}$, and a co-orderly $C L$-labeling $\lambda^{\prime \prime}$, such that any maximal chain $\mathbf{c}$ in $P$ has the same set of ascents and descents under each of the three labelings $\lambda, \lambda^{\prime}$, and $\lambda^{\prime \prime}$.

The proof involves constructing a recursive atom ordering from $\lambda$, and then constructing a $C L$-labeling with the desired properties from the recursive atom ordering.

Note 5.10. The result of Lemma 5.9 is not known to be true if ' $C L$ ' is replaced by ' $E L$ '.

To find a $C L$-labeling of $P_{1} \times P_{2}$, we label each edge in $P_{1} \times P_{2}$ with the edge in $P_{1}$ or $P_{2}$ to which it projects. More formally, notice that any rooted cover relation $(\mathbf{r}, x \lessdot y)$ projects to a cover relationship in one coordinate, and to a point in the other. Then the product labeling, denoted $\lambda_{1} \times \lambda_{2}$, labels $(\mathbf{r}, x \lessdot y)$ with $\lambda_{i}\left(p_{i}(\mathbf{r}), p_{i}(x \lessdot y)\right)$, where $i$ is the coordinate where projection is nontrivial. It is straightforward to show that $\lambda_{1} \times \lambda_{2}$ is a $C L$-labeling if $\lambda_{1}$ and $\lambda_{2}$ are $C L$-labelings of $P_{1}$ and $P_{2}$, and where we order $S_{\lambda_{1}} \cup S_{\lambda_{2}}$ by any shuffle of $S_{\lambda_{1}}$ and $S_{\lambda_{2}}$ [4, Proposition 10.15].

The idea behind finding a $C L$-labeling of $P_{1} \check{\times} P_{2}$ (or similarly $P_{1} \hat{\times} P_{2}$ ) is to restrict $\lambda_{1} \times \lambda_{2}$ to $P_{1} \check{\times} P_{2}$. For a cover relation $x \lessdot y$ where $x \neq \hat{0}$, this works very well, as $x \lessdot y$ 
in $P_{1} \check{\times} P_{2}$ is also a cover relation in $P_{1} \times P_{2}$, and the roots project straightforwardly. The problem comes at cover relations $\hat{0} \lessdot y$, which project to a cover relation in both $P_{1}$ and $P_{2}$. Here, we need to combine the labels $\lambda_{1}\left(\hat{0} \lessdot p_{1}(y)\right)$ and $\lambda_{2}\left(\hat{0} \lessdot p_{2}(y)\right)$.

The orderly labelings constructed in Lemma 5.9 are a tool to perform this combination in a manner that preserves the $C L$-property. Let $P_{1}$ and $P_{2}$ have orderly $C L$-labelings $\lambda_{1}$ and $\lambda_{2}$, with disjoint label sets $S_{1}$ and $S_{2}$. Suppose the label sets are shuffled together as

$$
S_{1}^{-}<S_{2}^{-}<S_{1}^{A}<S_{2}^{A}<S_{1}^{+}<S_{2}^{+} .
$$

Then the lower reduced product labeling $\lambda_{1} \check{\times} \lambda_{2}$ labels an edge $\hat{0} \lessdot y$ with the word $\lambda_{1}\left(p_{1}(\hat{0} \lessdot y)\right) \lambda_{2}\left(p_{2}(\hat{0} \lessdot y)\right)$ in $S_{1}^{A} S_{2}^{A}$ (lexicographically ordered), while all other rooted edges $(\mathbf{r}, x \lessdot y)$ (for $x \neq \hat{0})$ are labeled with the nontrivial projection $\lambda_{i}\left(p_{i}(\mathbf{r}), p_{i}(x \lessdot y)\right.$ ) as in $\lambda_{1} \times \lambda_{2}$. Björner and Wachs proved [4, Theorems 10.2 and 10.17] that $\lambda_{1} \check{\times} \lambda_{2}$ is a $C L$-labeling of $P_{1} \check{\times} P_{2}$.

Similarly, if $\lambda_{1}$ and $\lambda_{2}$ are co-orderly $C L$-labelings of $P_{1}$ and $P_{2}$, with disjoint label sets shuffled together as for the orderly labelings above, we define the upper reduced product labeling $\lambda_{1} \hat{\times} \lambda_{2}$ as follows. Label an edge of the form $(\mathbf{r}, x \lessdot \hat{1})$ with the word

$$
\lambda_{1}\left(p_{1}(\mathbf{r}), p_{1}(x \lessdot \hat{1})\right) \lambda_{2}\left(p_{2}(\mathbf{r}), p_{2}(x \lessdot \hat{1})\right)
$$

in $S_{1}^{A} S_{2}^{A}$, and all other edges $(\mathbf{r}, x \lessdot y)($ for $y \neq \hat{1})$ as in $\lambda_{1} \times \lambda_{2}$. Then $[4$, Theorems 10.2 and 10.17] gives us that $\lambda_{1} \hat{\times} \lambda_{2}$ is a $C L$-labeling of $P_{1} \hat{\times} P_{2}$.

Example 5.11. The labeling $\lambda_{\text {div }}$ we constructed for the $d$-divisible partition lattice was an co-orderly $E L$-labeling of the dual lattice: actually, $S^{A}$ was just $\{0\}$. As discussed in Lemma 3.5, intervals split as products, and the restriction of $\lambda_{\text {div }}$ to an interval splits as the appropriate product labeling.

We summarize in the following theorem:

Theorem 5.12. (Björner and Wachs [4, Proposition 10.15 and Theorem 10.17]) Let $P_{1}$ and $P_{2}$ be posets, with respective labelings $\lambda_{1}$ and $\lambda_{2}$.

1. If $\lambda_{1}$ and $\lambda_{2}$ are CL-labelings (EL-labelings), then $\lambda_{1} \times \lambda_{2}$ is a CL-labeling (ELlabeling) of $P_{1} \times P_{2}$.

2. If $\lambda_{1}$ and $\lambda_{2}$ are orderly CL-labelings, then $\lambda_{1} \check{\times} \lambda_{2}$ is a CL-labeling of $P_{1} \check{\times} P_{2}$.

3. If $\lambda_{1}$ and $\lambda_{2}$ are co-orderly CL-labelings, then $\lambda_{1} \hat{\times} \lambda_{2}$ is a CL-labeling of $P_{1} \hat{\times} P_{2}$.

\subsection{CL-ceds of product posets}

Fix our notation as in Section 5.2, but suppose in addition that $P_{1}$ and $P_{2}$ have $C L$-ceds $\left\{\Sigma_{s}^{(1)}\right\}$ and $\left\{\Sigma_{t}^{(2)}\right\}$ with respect to the $C L$-labelings $\lambda_{1}$ and $\lambda_{2}$. Denote the resulting ears of new chains as $\left\{\Delta_{s}^{(1)}\right\}$ and $\left\{\Delta_{t}^{(2)}\right\}$, as in Section 2.5. Then take $\Sigma_{s, t}$ to be the appropriate product of $\Sigma_{s}^{(1)}$ and $\Sigma_{t}^{(2)}$, and $\Delta_{s, t}$ to be the associated ear of new chains.

We first notice that there is no inconsistency with the notation used in Section 5.2: 
Lemma 5.13. A maximal chain $\mathbf{c}$ is in $\Delta_{s, t}$ if and only if $p_{1}(\mathbf{c})$ is in $\Delta_{s}^{(1)}$ and $p_{2}(\mathbf{c})$ is in $\Delta_{t}^{(2)}$.

Proof. The statement follows straightforwardly from the fact that the maximal chains of $\Sigma_{s, t}$ are those that project to $\Sigma_{s}^{(1)}$ and $\Sigma_{t}^{(2)}$.

As we did in Section 5.2, order the $\left\{\Sigma_{s, t}\right\}$ according to the lexicographic order of $(s, t)$. Let $\lambda$ be the appropriate product $C L$-labeling, where we assume without loss of generality via Lemma 5.9 that $\lambda_{1}$ and $\lambda_{2}$ are orderly or co-orderly. Then we will prove:

Theorem 5.14. $\left\{\Sigma_{s, t}\right\}$ is a $C L$-ced for $|P|$ with respect to $\lambda$.

Proof. Proposition 5.1 tells us that (CLced-polytope) is satisfied, and (CLced-union) is immediate from the definitions.

For (CLced-bdry), we work backwards, and notice that we have already shown in

Theorem 5.7 that $\partial \Delta_{s, t}=\Delta_{s, t} \cap\left(\bigcup_{u, v<s, t} \Delta_{u, v}\right)$. Lemma 2.8 meanwhile gives that $\partial \Delta_{s, t}=$ $\Delta_{s, t} \cap \overline{\left|\Sigma_{s, t}\right| \backslash \Delta_{s, t}}$, hence that a chain $\mathbf{c}$ with extensions in both $\Delta_{s, t}$ and $\Delta_{u, v}$ has an extension in $\mathcal{M}\left(\Sigma_{s, t}\right) \backslash \mathcal{M}\left(\Delta_{s, t}\right)$, as required. (A direct proof is also straightforward.)

It remains to check (CLced-desc). Although the statement of this property is very similar to [4, Theorem 10.17] (which says that $\lambda$ is a $C L$-labeling), the proof in [4] uses some machinery. So we work from scratch, as follows.

If $P=P_{1} \times P_{2}$ and we are considering the rooted interval $[x, y]_{\mathbf{r}}$, then the labels of a maximal chain $\mathbf{c}_{\mathbf{r}}$ on $[x, y]_{\mathbf{r}}$ are the same as the labels of $p_{1}(\mathbf{c})_{p_{1}(\mathbf{r})}$ union with the labels of $p_{2}(\mathbf{c})_{p_{2}(\mathbf{r})}$, "shuffled together" in some order. Thus, if $\mathbf{c}_{\mathbf{r}}$ is descending, then the projections must also be descending. Since the label sets $S_{1}$ and $S_{2}$ are taken to be disjoint, there is a unique way of shuffling the two label sets (and so the two chains) together to get a descending chain.

For $P=P_{1} \check{\times} P_{2}$, the proof is the same unless $x=\hat{0}$. In this case, the first label of a maximal chain c is in $S_{1}^{A} S_{2}^{A}$, while the first label of the projections are in $S_{1}^{A}$ and $S_{2}^{A}$, respectively. If $\mathbf{c}$ is descending, then all labels after the first are from $S_{1}^{-}$or $S_{2}^{-}$, since $S_{1}^{-}<S_{2}^{-}<S_{1}^{A} S_{2}^{A}<S_{1}^{+}<S_{2}^{+}$, and thus $p_{1}(\mathbf{c})$ and $p_{2}(\mathbf{c})$ are descending, and we argue as before.

The proof for $P=P_{1} \hat{\times} P_{2}$ is entirely similar to that for $P_{1} \check{\times} P_{2}$.

\section{$6 \quad$ Further questions}

The close relationship between the techniques used in Sections 3 and 4 leads us to ask the following question.

Question 1. Are there other families of posets with similar structure to $\Pi_{n}^{d}$ and $\mathfrak{C}(G)$ ? Can the techniques used in Sections 3 and 4 be used to construct dual EL-labelings and EL-ceds? 
What we mean by 'similar' here is not clear. At the least, we need a poset $P$ where every interval of the form $[a, \hat{1}]$ is supersolvable, and where the supersolvable structure is canonically determined, i.e., such that we can label all edges of $P \backslash\{\hat{0}\}$ in a way that restricts to a supersolvable labeling on each such $[a, \hat{1}]$ interval. We then need a way to sign the edges giving an EL-labeling, and the poset has to somehow be 'wide' or 'rich' enough to have an $E L$-ced.

One possible source of such examples is the theory of exponential structures. An exponential structure is a family of posets with each upper interval isomorphic to the partition lattice, and each lower interval isomorphic to a product of smaller elements in the same family. Exponential structures were introduced in [27], where the family of $d$-divisible partition lattices was shown to be one example. Shellings are constructed for some other examples in [21,35].

Question 2. Can techniques like those used in Section 3 (and Section 4) be used to construct dual $E L$-labelings and/or $E L$-ceds of exponential structures besides the $d$-divisible partition lattice?

However, it is not a priori clear how to construct a labeling that restrict to a supersolvable labeling on any $[a, \hat{1}]$ for exponential structures. In examples even finding an $E L$-labeling often seems to be a difficult problem.

A question suggested by the results of Section 5 is:

Question 3. Are there other operations on posets that preserve convex ear decompositions and/or $C L$-ceds?

For example, Schweig shows [23, Theorem 5.1] that rank-selected supersolvable and geometric lattices have convex ear decompositions. Do all rank-selected subposets of posets with convex ear decompositions have a convex ear decomposition? Are there any other useful constructions that preserve having a convex ear decomposition and/or $E L$ ced? A place to start looking would be in Björner and Wach's papers [2, 3, 4], where they answer many such questions for $E L / C L$-labelings.

\section{Acknowledgements}

Thanks to Ed Swartz for introducing me to convex ear decompositions; and to him, Jay Schweig, and my graduate school advisor Ken Brown for many helpful discussions about them. Tom Rishel listened to and commented on many intermediate versions of the results

and definitions of this paper. Sam Hsiao helped me in understanding the material of Proposition 5.1, and in looking for its extension to Lemma 1.5. Vic Reiner pointed out that the labeling based on pivots of $\mathfrak{C}(G)$ that I used in [36] was really a supersolvable labeling, which suggested the improved EL-labeling used in Section 4. Volkmar Welker suggested exponential structures as a possible area for further exploration. The anonymous referee gave many helpful comments. 


\section{References}

[1] Anders Björner, Shellable and Cohen-Macaulay partially ordered sets, Trans. Amer. Math. Soc. 260 (1980), no. 1, 159-183.

[2] Anders Björner and Michelle L. Wachs, On lexicographically shellable posets, Trans. Amer. Math. Soc. 277 (1983), no. 1, 323-341.

[3] Shellable nonpure complexes and posets. I, Trans. Amer. Math. Soc. 348 (1996), no. 4, 1299-1327.

[4] _ Shellable nonpure complexes and posets. II, Trans. Amer. Math. Soc. 349 (1997), no. 10, 3945-3975.

[5] Kenneth S. Brown, The coset poset and probabilistic zeta function of a finite group, J. Algebra 225 (2000), no. 2, 989-1012.

[6] Manoj K. Chari, Two decompositions in topological combinatorics with applications to matroid complexes, Trans. Amer. Math. Soc. 349 (1997), no. 10, 3925-3943.

[7] Gopal Danaraj and Victor Klee, Shellings of spheres and polytopes, Duke Math. J. 41 (1974), 443-451.

[8] G. Ewald and G. C. Shephard, Stellar subdivisions of boundary complexes of convex polytopes, Math. Ann. 210 (1974), 7-16.

[9] Günter Ewald, Combinatorial convexity and algebraic geometry, Graduate Texts in Mathematics, vol. 168, Springer-Verlag, New York, 1996.

[10] The GAP Group, GAP - Groups, Algorithms, and Programming, Version 4.4.10, 2007, (http://www.gap-system.org/ ).

[11] Kenkichi Iwasawa, Über die endlichen Gruppen und die Verbände ihrer Untergruppen, J. Fac. Sci. Imp. Univ. Tokyo. Sect. I. 4 (1941), 171-199.

[12] Gil Kalai, A new basis of polytopes, J. Combin. Theory Ser. A 49 (1988), no. 2, 191-209.

[13] Jon McCammond, Noncrossing partitions in surprising locations, Amer. Math. Monthly 113 (2006), no. 7, 598-610, arXiv:math/0601687.

[14] Peter McNamara, EL-labelings, supersolvability and 0-Hecke algebra actions on posets, J. Combin. Theory Ser. A 101 (2003), no. 1, 69-89, arXiv:math/0111156.

[15] Peter McNamara and Hugh Thomas, Poset edge-labellings and left modularity, European Journal of Combinatorics 27 (2006), no. 1, 101-113, arXiv:math.CO/0211126.

[16] James Munkres, Topology, second ed., Prentice Hall, Englewood Cliffs, N.J., 2000.

[17] Kathryn Nyman and Ed Swartz, Inequalities for the $h$ - and flag h-vectors of geometric lattices, Discrete and Computational Geometry 32 (2004), no. 4, 533-548, arXiv:math.CO/0405535.

[18] Daniel Quillen, Homotopy properties of the poset of nontrivial p-subgroups of a group, Adv. in Math. 28 (1978), no. 2, 101-128. 
[19] Daniel A. Ramras, Connectivity of the coset poset and the subgroup poset of a group, J. Group Theory 8 (2005), no. 6, 719-746, arXiv:math/0210001.

[20] C. P. Rourke and B. J. Sanderson, Introduction to piecewise-linear topology, SpringerVerlag, New York, 1972, Ergebnisse der Mathematik und ihrer Grenzgebiete, Band 69.

[21] Bruce E. Sagan, Shellability of exponential structures, Order 3 (1986), no. 1, 47-54.

[22] Roland Schmidt, Subgroup lattices of groups, de Gruyter Expositions in Mathematics, vol. 14, Walter de Gruyter \& Co., Berlin, 1994.

[23] Jay Schweig, Several convex ear decompositions, 2006, arXiv:math/0605238.

[24] John Shareshian, On the shellability of the order complex of the subgroup lattice of a finite group, Trans. Amer. Math. Soc. 353 (2001), no. 7, 2689-2703 (electronic).

[25] Rodica Simion, Noncrossing partitions, Discrete Math. 217 (2000), no. 1-3, 367-409, Formal power series and algebraic combinatorics (Vienna, 1997).

[26] R. P. Stanley, Supersolvable lattices, Algebra Universalis 2 (1972), 197-217.

[27] Richard P. Stanley, Exponential structures, Stud. Appl. Math. 59 (1978), no. 1, 7382.

[28] _ Combinatorics and commutative algebra, second ed., Progress in Mathematics, vol. 41, Birkhäuser Boston Inc., Boston, MA, 1996.

[29] - The descent set and connectivity set of a permutation, J. Integer Seq. 8 (2005), no. 3, Article 05.3.8, 9 pp. (electronic), arXiv:math/0507224.

[30] Sheila Sundaram, Applications of the Hopf trace formula to computing homology representations, Jerusalem combinatorics '93, Contemp. Math., vol. 178, Amer. Math. Soc., Providence, RI, 1994, pp. 277-309.

[31] Ed Swartz, g-elements, finite buildings and higher Cohen-Macaulay connectivity, J. Combin. Theory Ser. A 113 (2006), no. 7, 1305-1320, arXiv:math/0512086.

[32] Jacques Thévenaz, The top homology of the lattice of subgroups of a soluble group, Discrete Math. 55 (1985), no. 3, 291-303.

[33] Michelle L. Wachs, A basis for the homology of the d-divisible partition lattice, Adv. Math. 117 (1996), no. 2, 294-318.

[34] James W. Walker, Canonical homeomorphisms of posets, European J. Combin. 9 (1988), no. 2, 97-107.

[35] Volkmar Welker, Direct sum decompositions of matroids and exponential structures, J. Combin. Theory Ser. B 63 (1995), no. 2, 222-244.

[36] Russ Woodroofe, Shelling the coset poset, J. Combin. Theory Ser. A 114 (2007), no. 4, 733-746, arXiv:math/0306346.

[37] _ An EL-labeling of the subgroup lattice, Proc. Amer. Math. Soc. 136 (2008), no. 11, 3795-3801, arXiv:0708.3539. 